\title{
Patterns of flavor signals in supersymmetric models
}

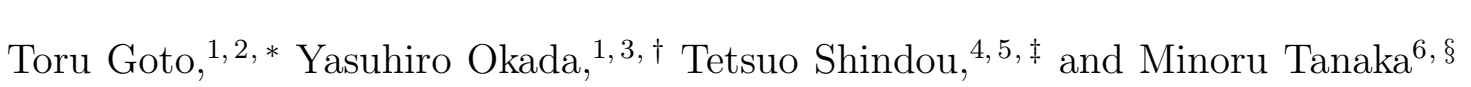 \\ ${ }^{1}$ Theory Group, KEK, Tsukuba, Ibaraki 305-0801, Japan \\ ${ }^{2}$ YITP, Kyoto University, Kyoto 606-8502, Japan \\ ${ }^{3}$ Department of Particle and Nuclear Physics, \\ The Graduate University for Advanced Studies \\ (Sokendai), Tsukuba, Ibaraki 305-0801, Japan \\ ${ }^{4}$ DESY Theory Group, Notkestrasse 85 D22607 Hamburg, Germany \\ ${ }^{5}$ SISSA/ISAS, via Beirut 2-4 I-34014 Trieste, Italy \\ ${ }^{6}$ Department of Physics, Graduate School of Science, \\ Osaka University, Toyonaka, Osaka 560-0043, Japan
}

(Dated: November 19, 2007)

\begin{abstract}
Quark and lepton flavor signals are studied in four supersymmetric models, namely the minimal supergravity model, the minimal supersymmetric standard model with right-handed neutrinos, $\mathrm{SU}(5)$ supersymmetric grand unified theory with right-handed neutrinos and the minimal supersymmetric standard model with $\mathrm{U}(2)$ flavor symmetry. We calculate $b \rightarrow s(d)$ transition observables in $B_{d}$ and $B_{s}$ decays, taking the constraint from the $B_{s}-\bar{B}_{s}$ mixing recently observed at Tevatron into account. We also calculate lepton flavor violating processes $\mu \rightarrow e \gamma, \tau \rightarrow \mu \gamma$ and $\tau \rightarrow e \gamma$ for the models with right-handed neutrinos. We investigate possibilities to distinguish the flavor structure of the supersymmetry breaking sector with use of patterns of various flavor signals which are expected to be measured in experiments such as MEG, LHCb and a future Super $B$ Factory.
\end{abstract}

PACS numbers: $12.60 . J v, 14.40 . \mathrm{Nd}, 12.15 . \mathrm{Hh}, 11.30 . \mathrm{Er}$

*Electronic address: tgoto@post.kek.jp

†Electronic address: yasuhiro.okada@kek.jp

‡Electronic address: tetsuo.shindou@desy.de

§Electronic address: tanaka@phys.sci.osaka-u.ac.jp

ฯPresent address. 


\section{INTRODUCTION}

The problem of flavors is one of the interesting aspects of particle physics. Results obtained at $B$ factory experiments so far indicate that the Cabibbo-Kobayashi-Maskawa (CKM) mixing [1] is the main mechanism of flavor mixing phenomena in the quark sector, although there still remains some room for new physics beyond the standard model (SM). On the other hand, in the lepton sector, neutrino experiments unveil large flavor mixings quite different from the quark sector [2, 3, 4, 5]. These mixings in the lepton sector are certainly beyond the SM, suggesting a new mechanism of flavor mixing. It is clear that flavor physics is a clue to new physics.

In the coming years, we expect new experimental results from the energy frontier, that is, CERN Large Hadron Collider (LHC) [6]. LHC experiments will provide us with invaluable information on new physics. Among several candidates of new physics, supersymmetry (SUSY) is the most attractive and widely discussed [7]. It is possible that some of the superparticles are discovered in the early stage of LHC experiments.

One of the key questions in realistic SUSY models is to identify the mechanism of SUSY breaking. The SUSY breaking mechanism can be explored by determining the SUSY mass spectrum in LHC experiments at the energy frontier. On the other hand, the whole flavor structure of the SUSY breaking cannot be determined by the energy frontier experiment alone. There is no a priori reason to exclude flavor changing soft SUSY breaking terms in the squark and the slepton sectors, and some of them are already strongly restricted by the existing low energy experimental data [8, 9]. It means that we can extract important aspects of the SUSY breaking mechanism from flavor physics.

Two new flavor experiments are under construction, and several others are proposed. The MEG experiment [10], which intends to search for the lepton flavor violating (LFV) process $\mu \rightarrow e \gamma$ at a branching ratio down to $10^{-13}$, will start data taking soon. The $\mathrm{LHCb}$ experiment [11, 12] is another dedicated flavor experiment under construction and will be ready by the LHC startup in 2008. It is designed to observe several rare decays and CP violations in $B$ and $B_{s}$ decays. There are plans of future Super $B$ Factories under discussion [13, 14, 15]. In addition to measure several $B$ decay observables with higher statistics, it is expected to search for LFV processes in tau decays at a branching ratio of $10^{-9}$. These new flavor experiments themselves and their interplay with LHC experiments at the energy 
frontier will augment our knowledge on flavors and eventually new physics.

Several strategies are possible in order to study the implication of the past and present experimental data on SUSY models and predictions of flavor signals in future experiments. One of them is a model-independent method based on the mass insertion [16, 17, 18]. In this approach, a general set of off-diagonal matrix elements (mass insertions) of the squarks and the sleptons is assumed, and one (or two) of the elements is (are) activated in order to obtain a bound from a specific experiment. Repeating this procedure for every relevant experiment, a list of bounds for the possible mass insertions is obtained. This list is used to evaluate flavor signals in future experiments. As an opposite way, a model specific analysis is possible [19, 20, 21, 22, 23, 24, 25]. In this approach, one specifies a SUSY model with a well-defined SUSY breaking sector and analyzes one (or more) selected flavor signal(s). In this way one can make definite predictions on observable quantities in flavor changing processes provided that the relevant model parameters are given.

In our previous works [26, 27], we adopted a different approach. We selected three wellmotivated SUSY models: the minimal supergravity (mSUGRA), the SU(5) SUSY grand unified theory (GUT) with right-handed neutrinos, and the minimal supersymmetric standard model (MSSM) with U(2) flavor symmetry. Each of these models has a distinct flavor structure in its SUSY breaking sector at the electroweak scale. Then, we investigated various flavor signals in these models in a unified fashion. This approach allows us to evaluate flavor signals definitely and to discuss the possibility to distinguish several different flavor structures in the SUSY breaking sector in future flavor experiments. The quark flavor signals which we studied are the CP violation parameter $\varepsilon_{K}$ in the $K^{0}-\bar{K}^{0}$ mixing, the $B_{d}-\bar{B}_{d}$ and the $B_{s}-\bar{B}_{s}$ mass splittings $\left(\Delta m_{B_{d}}\right.$ and $\Delta m_{B_{s}}$ respectively), CP asymmetries in $B \rightarrow J / \psi K_{S}$ and related modes, the direct and the mixing-induced CP asymmetries in $b \rightarrow s \gamma$, and the $\mathrm{CP}$ asymmetry in $B \rightarrow \phi K_{S}$. An LFV process $\mu \rightarrow e \gamma$ was studied in addition. Comparing predictions of the models with each other, we showed that the study of quark flavor signals at low energies could discriminate several SUSY models that have different flavor structures in their SUSY breaking sectors.

In the present work, we extend our previous works. New features and improvements of the present work are the followings.

(i) In addition to the three models, we consider the MSSM with right-handed neutrinos and the seesaw mechanism without GUT. 
(ii) Three types of Ansätze for the neutrino Yukawa coupling matrix are studied.

(iii) New and up-to-date experimental data are incorporated. In particular $\Delta m_{B_{s}}$ measured by the CDF and D $\varnothing$ experiments at Fermilab Tevatron affects predictions of several $B$ decay modes [18, 28].

(iv) LFV tau decays and their implications are examined.

(v) As computational improvements, two-loop renormalization group equations for the MSSM (with right-handed neutrinos) parameter running and one-loop threshold corrections at the electroweak scale are implemented.

With these new features and improvements, we pursue the possibility to distinguish the flavor structure of the SUSY breaking sector by low energy flavor experiments and to understand the SUSY breaking mechanism consequently.

A brief summary of our analysis is as follows. We expect significant flavor signals in the lepton sector for the models with right-handed neutrinos. In the MSSM with right-handed neutrinos, depending on the texture of the neutrino Yukawa coupling matrix, one of the LFV processes, $\mu \rightarrow e \gamma, \tau \rightarrow \mu \gamma$ and $\tau \rightarrow e \gamma$ could be discovered in near future. In the SU(5) SUSY GUT with right-handed neutrinos, in addition to the above texture dependent signals, $\mu \rightarrow e \gamma$ can be close to the present experimental bound due to GUT interactions. As for the quark flavor signals, $\mathrm{CP}$ violating asymmetries in $b \rightarrow s$ and $b \rightarrow d$ transitions can be significant in the SU(5) SUSY GUT with right-handed neutrinos and in the U(2) model. Enhanced modes vary according to the texture of the neutrino Yukawa coupling matrix in the SU(5) SUSY GUT with right-handed neutrinos. Our analysis indicates that clarifying a pattern of the quark and lepton flavor signals is an important step to determine the correct SUSY model.

This paper is organized as follows. In Sec. II the models are presented and the relevant SUSY parameters are introduced. Our numerical analysis with the experimental inputs and the outline of computational procedure are shown in Sec. III. Conclusions are given in Sec. IV. 


\section{MODELS AND SUSY PARAMETERS}

\section{A. Models}

In this section, we give a brief description of the models considered in this paper. They are well-motivated examples of SUSY models, and are chosen as representatives that have distinct flavor signals. Every model is reduced to the MSSM at low energy scale, which is an $\mathrm{SU}(3)_{C} \times \mathrm{SU}(2)_{L} \times \mathrm{U}(1)_{Y}$ supersymmetric gauge theory with the SUSY being softly broken. The MSSM matter contents are the following chiral superfields:

$$
\begin{array}{lll}
Q_{i}\left(3,2, \frac{1}{6}\right), & \bar{U}_{i}\left(\overline{3}, 1,-\frac{2}{3}\right), & \bar{D}_{i}\left(\overline{3}, 1, \frac{1}{3}\right), \\
L_{i}\left(1,2,-\frac{1}{2}\right), & \bar{E}_{i}(1,1,1), \quad(i=1,2,3) \\
H_{1}\left(1,2,-\frac{1}{2}\right), & H_{2}\left(1,2, \frac{1}{2}\right),
\end{array}
$$

where the gauge quantum numbers are shown in parentheses. The MSSM superpotential can be written as

$$
\mathcal{W}_{\mathrm{MSSM}}=y_{D}^{i j} \bar{D}_{i} Q_{j} H_{1}+y_{U}^{i j} \bar{U}_{i} Q_{j} H_{2}+y_{E}^{i j} \bar{E}_{i} L_{j} H_{1}+\mu H_{1} H_{2}
$$

with an assumption of $R$-parity conservation and renormalizability. The SUSY breaking effect is described by the following soft SUSY breaking terms in the Lagrangian.

$$
\begin{aligned}
-\mathcal{L}_{\mathrm{soft}}^{\mathrm{MSSM}}= & \left(m_{Q}^{2}\right)^{i j} \tilde{q}_{i}^{\dagger} \tilde{q}_{j}+\left(m_{U}^{2}\right)^{i j} \tilde{u}_{i}^{\dagger} \tilde{u}_{j}+\left(m_{D}^{2}\right)^{i j} \tilde{d}_{i}^{\dagger} \tilde{d}_{j}+\left(m_{L}^{2}\right)^{i j} \tilde{l}_{i}^{\dagger} \tilde{l}_{j}+\left(m_{E}^{2}\right)^{i j} \tilde{e}_{i}^{\dagger} \tilde{e}_{j} \\
& +m_{H_{1}}^{2} h_{1}^{\dagger} h_{1}+m_{H_{2}}^{2} h_{2}^{\dagger} h_{2}-\left(B \mu h_{1} h_{2}+\text { H.c. }\right) \\
& +\left(A_{U}^{i j} \tilde{u}_{i}^{\dagger} \tilde{q}_{j} h_{2}+A_{D}^{i j} \tilde{d}_{i}^{\dagger} \tilde{q}_{j} h_{1}+A_{E}^{i j} \tilde{e}_{i}^{\dagger} \tilde{l}_{j} h_{1}+\text { H.c. }\right) \\
& +\frac{M_{3}}{2} \overline{\tilde{g}} \tilde{g}+\frac{M_{2}}{2} \tilde{\bar{W}} \tilde{W}+\frac{M_{1}}{2} \overline{\tilde{B}} \tilde{B},
\end{aligned}
$$

where $\tilde{q}_{i}, \tilde{u}_{i}^{\dagger}, \tilde{d}_{i}^{\dagger}, \tilde{l}_{i}, \tilde{e}_{i}^{\dagger}, h_{1}$, and $h_{2}$ are the corresponding scalar components of the chiral superfields given in Eq. (1), and $\tilde{g}, \tilde{W}$, and $\tilde{B}$ are $\mathrm{SU}(3)_{C}, \mathrm{SU}(2)_{L}$ and $\mathrm{U}(1)_{Y}$ gauge fermions, respectively.

\section{The minimal supergravity model}

The mSUGRA consists of the MSSM sector and a hidden sector where the SUSY is assumed to be spontaneously broken. Only a gravitational interaction interconnects these 
two sectors. This gravitational interaction mediates the SUSY breaking effect from the hidden sector to the observable MSSM sector, and the soft breaking terms in Eq. (3) are induced in the following manner:

$$
\begin{aligned}
& \left(m_{Q}^{2}\right)_{i j}=\left(m_{U}^{2}\right)_{i j}=\left(m_{D}^{2}\right)_{i j}=\left(m_{L}^{2}\right)_{i j}=\left(m_{E}^{2}\right)_{i j}=m_{0}^{2} \delta_{i j} \\
& m_{H_{1}}^{2}=m_{H_{2}}^{2}=m_{0}^{2} \\
& A_{U}^{i j}=m_{0} A_{0} y_{U}^{i j}, \quad A_{D}^{i j}=m_{0} A_{0} y_{D}^{i j}, \quad A_{E}^{i j}=m_{0} A_{0} y_{E}^{i j} \\
& M_{1}=M_{2}=M_{3}=m_{1 / 2}
\end{aligned}
$$

where we assume the GUT relation among the gaugino masses. The above relations are applied at the energy scale where the soft breaking terms are induced by the gravitational interaction. We identify this scale with the GUT scale $\left(\mu_{G} \simeq 2 \times 10^{16} \mathrm{GeV}\right)$ for simplicity. Thus the soft breaking terms are specified at $\mu_{G}$ by the universal scalar mass, $m_{0}$, the universal gaugino mass, $m_{1 / 2}$, and the universal trilinear coupling, $A_{0}$. The soft breaking terms at the electroweak scale are determined by solving renormalization group equations.

In this model, the only source of flavor mixings is the CKM matrix. New flavor mixings in the squark sector at the electroweak scale come from the CKM matrix through radiative corrections [19, 20]. In addition to the CP phase in the CKM matrix, there can be two physically independent CP phases. We take the complex phase of the $\mu$ term $\left(\phi_{\mu} \equiv \arg \mu\right)$ and the phase of $A_{0}\left(\phi_{A} \equiv \arg A_{0}\right)$ as the new CP phases while we take the gaugino mass $m_{1 / 2}$ as real and positive by convention. These CP phases contribute to the neutron and electron electric dipole moments (EDMs) [9, 29, 30, 31, 32] and experimental constraints on these phases are very severe.

We assume that the generation mechanism of neutrino masses in this model does not affect the flavor mixing in the SUSY sector. For example, in the mSUGRA model with right-handed neutrinos, which is described bellow, the effect of the neutrino mass on the flavor mixing in the SUSY sector is negligible in a small right-handed neutrino mass limit.

\section{The MSSM with right-handed neutrinos}

Recent developments of neutrino experiments have established the existence of small finite masses of neutrinos. A simple extension of the mSUGRA model for giving small finite masses of neutrinos is introducing gauge singlet right-handed Majorana neutrino superfields, 
$\bar{N}_{i}(i=1,2,3)$. This is known as the type I seesaw mechanism [34]. The superpotential can be written as

$$
\mathcal{W}_{\mathrm{MSSM} \nu_{R}}=\mathcal{W}_{\mathrm{MSSM}}+\left(y_{N}\right)^{i j} \bar{N}_{i} L_{j} H_{2}+\frac{1}{2}\left(M_{N}\right)^{i j} \bar{N}_{i} \bar{N}_{j}
$$

which leads to the following higher dimensional term

$$
\Delta \mathcal{W}_{\nu}=-\frac{1}{2} K_{\nu}^{i j}\left(L_{i} H_{2}\right)\left(L_{j} H_{2}\right), \quad K_{\nu}=\left(y_{N}^{T}\right)^{i k}\left(M_{N}^{-1}\right)^{k l}\left(y_{N}\right)^{l j}
$$

after heavy fields $\bar{N}_{1,2,3}$ are integrated out at the energy scale below the Majorana mass scale $\left(\equiv \mu_{R}\right)$. This higher dimensional term yields the neutrino mass matrix by the electroweak symmetry breaking as

$$
\left(m_{\nu}\right)^{i j}=\left(K_{\nu}\right)^{i j}\left\langle h_{2}\right\rangle^{2}
$$

Taking the basis in which the charged lepton mass matrix is diagonal, one can obtain the observable neutrino mass eigenvalues and the Pontecorvo-Maki-Nakagawa-Sakata (PMNS) mixing matrix [35] as

$$
\left(m_{\nu}\right)^{i j}=\left(V_{\mathrm{PMNS}}^{*}\right)^{i k} m_{\nu_{k}}\left(V_{\mathrm{PMNS}}^{\dagger}\right)^{k j}
$$

As for the soft breaking terms, scalar mass terms, $A$ terms and $B$ terms of sneutrinos, $\tilde{\nu}_{i}^{\dagger}$, are added as

$$
-\mathcal{L}_{\mathrm{soft}}^{\mathrm{MSSM} \nu_{R}}=-\mathcal{L}_{\mathrm{soft}}^{\mathrm{MSSM}}+\left(m_{N}^{2}\right)^{i j} \tilde{\nu}_{i}^{\dagger} \tilde{\nu}_{j}+\left(A_{N}^{i j} \tilde{\nu}_{i} \tilde{l}_{j} h_{2}+\left(\tilde{m}_{N}^{2}\right)^{i j} \tilde{\nu}_{i}^{\dagger} \tilde{\nu}_{j}^{\dagger}+\text { H.c. }\right)
$$

We assume that the soft breaking terms are generated in a universal fashion at $\mu_{G}$, i.e.

$$
\left(m_{N}^{2}\right)^{i j}=m_{0}^{2} \delta^{i j}, \quad A_{N}^{i j}=m_{0} A_{0} y_{N}^{i j} .
$$

We neglect the $\tilde{m}_{N}^{2}$ terms in the present work. These terms can significantly affect the EDMs, while contributions to the lepton flavor violation processes are sub-dominant [36].

The new flavor mixing in the scalar lepton sector comes from the neutrino mixing through the renormalization group running between $\mu_{G}$ and $\mu_{R}$. In the leading logarithmic approximation, they are given as

$$
\begin{aligned}
& \left(m_{L}^{2}\right)^{i j} \simeq-\frac{1}{8 \pi^{2}} m_{0}^{2}\left(3+\left|A_{0}\right|^{2}\right)\left(y_{N}^{\dagger} y_{N}\right)^{i j} \ln \frac{\mu_{G}}{\mu_{R}} \\
& \left(m_{E}^{2}\right)^{i j} \simeq 0 \\
& \left(A_{E}\right)^{i j} \simeq-\frac{3}{8 \pi^{2}} m_{0} A_{0} y_{e}^{i}\left(y_{N}^{\dagger} y_{N}\right)^{i j} \ln \frac{\mu_{G}}{\mu_{R}}
\end{aligned}
$$


for $i \neq j$. We numerically solve full RGEs in the actual analysis given in Sec. III. Consequences of these mixings on lepton flavor violating processes have been investigated from various aspects. Lepton flavor violating processes such as $\mu \rightarrow e \gamma$ are sensitive to the off-diagonal elements of $y_{N}^{\dagger} y_{N}$ [37].

As we have discussed in the end of the last subsection, this model reduces to the mSUGRA model in the limit of $y_{N} \rightarrow 0$. For instance, the effect of (11) is negligible if $\mu_{R} \ll 10^{12} \mathrm{GeV}$.

We consider three typical structures of the neutrino Yukawa couplings.

- degenerate case

$$
y_{N}=\frac{\sqrt{M_{N}}}{\left\langle h_{2}\right\rangle}\left(\begin{array}{ccc}
\sqrt{m_{\nu_{1}}} & 0 & 0 \\
0 & \sqrt{m_{\nu_{2}}} & 0 \\
0 & 0 & \sqrt{m_{\nu_{3}}}
\end{array}\right) V_{\mathrm{PMNS}}^{\dagger} .
$$

This is a case that all the masses of the right-handed neutrinos are the same and there are no CP phases in the heavy neutrino sector. In Eq. (12), $M_{N}$ denotes the eigenvalue of the right-handed neutrino mass matrix, i.e. $\left(M_{N}\right)^{i j}=M_{N} \delta^{i j}$. In this simplest case, the mixing in $y_{N}$ should be identified with the PMNS mixing because there is no flavor structure in $M_{N}$. The large mixing in the PMNS matrix leads to large off-diagonal elements of $y_{N}^{\dagger} y_{N}$, which enhance the $\mu \rightarrow e \gamma$ branching ratio. Therefore the SUSY breaking parameter space is strongly constrained by the present experimental limit.

- non-degenerate (I)

$$
y_{N}=\left(\begin{array}{ccc}
y_{11} & 0 & 0 \\
0 & y_{22} & y_{23} \\
0 & y_{32} & y_{33}
\end{array}\right)
$$

In this case, the PMNS mixing arises from the above $y_{N}$ and a non-degenerate mass matrix of right-handed neutrinos, $M_{N}$, as is described in Sec. IIIB. Because $y_{N}^{\dagger} y_{N}$ has the same texture as $y_{N}$ in Eq. (13),$\mu \rightarrow e \gamma$ is suppressed enough to satisfy the present experimental bound. As for other LFV processes, $\tau \rightarrow e \gamma$ is also suppressed, while $\tau \rightarrow \mu \gamma$ is not. The specific structure in Eq. (13) could be an implication of electronnumber conservation which works above the right-handed neutrino mass scale, $\mu_{R}$, and is broken by the right-handed neutrino mass matrix, $M_{N}$. 
- non-degenerate (II)

$$
y_{N}=\left(\begin{array}{ccc}
y_{11} & 0 & y_{13} \\
0 & y_{22} & 0 \\
y_{31} & 0 & y_{33}
\end{array}\right)
$$

This case is similar to the non-degenerate (I) case, except that the first and the second generations are interchanged in $y_{N}$. Accordingly, $\mu \rightarrow e \gamma$ and $\tau \rightarrow \mu \gamma$ are suppressed, while we expect a larger branching ratio of $\tau \rightarrow e \gamma$.

\section{The SU(5) SUSY GUT with right-handed neutrinos}

The idea of supersymmetric grand unification is supported by the precise determination of three gauge coupling constants at LEP and other experiments in the last decade. In view of this, we consider SU(5) SUSY GUT with right-handed neutrinos as an extension of the MSSM with right-handed neutrinos. Here we follow the analysis of Ref. [22, 26] and we give a brief description of the model.

This model is defined by the following superpotential:

$$
\begin{aligned}
\mathcal{W}_{\mathrm{SU}(5) \nu_{R}}= & \frac{1}{8} \epsilon_{a b c d e}\left(\lambda_{U}\right)^{i j}\left(T_{i}\right)^{a b}\left(T_{j}\right)^{c d} H^{e}+\left(\lambda_{D}\right)^{i j}\left(\bar{F}_{i}\right)_{a}\left(T_{j}\right)^{a b} \bar{H}_{b} \\
& +\left(\lambda_{N}\right)^{i j} \bar{N}_{i}\left(\bar{F}_{j}\right)_{a} H^{a}+\frac{1}{2}\left(M_{N}\right)^{i j} \bar{N}_{i} \bar{N}_{j}+\mathcal{W}_{H}+\Delta \mathcal{W}_{\mathrm{SU}(5) \nu_{R}},
\end{aligned}
$$

where $i$ and $j$ are generation indexes, while $a, b, c, d$ and $e$ are $\mathrm{SU}(5)$ indices. $\epsilon_{a b c d e}$ denotes the totally antisymmetric tensor of the $\mathrm{SU}(5) . T_{i}, \bar{F}_{i}$, and $\bar{N}_{i}$ are $\mathbf{1 0}, \overline{\mathbf{5}}$, and $\mathbf{1}$ representations of the $\mathrm{SU}(5)$ gauge group, respectively. $Q_{i}, \bar{U}_{i}$, and $\bar{E}_{i}$ are embedded in $T_{i}, \bar{F}_{i}$ consists of $\bar{D}_{i}$ and $L_{i}$, and $\bar{N}_{i}$ is identified with the right-handed heavy Majorana neutrinos. $H(\bar{H})$ denotes a Higgs superfield in $\mathbf{5}(\overline{\mathbf{5}})$ representation and includes $H_{C}\left(\mathbf{3}, \mathbf{1},-\frac{1}{3}\right)$ and $H_{2}\left(\bar{H}_{C}\left(\overline{\mathbf{3}}, \mathbf{1}, \frac{1}{3}\right)\right.$ and $\left.H_{1}\right) .\left(\lambda_{U}\right)^{i j},\left(\lambda_{D}\right)^{i j}$, and $\left(\lambda_{N}\right)^{i j}$ are the Yukawa coupling matrices, and $\left(M_{N}\right)^{i j}$ is the Majorana mass matrix. The superpotential for Higgs superfields, $\mathcal{W}_{H}$, contains terms with $H, \bar{H}$, and $\Sigma_{b}^{a}$ which is a 24 representation of the $\mathrm{SU}(5)$ gauge group. It is assumed that a vacuum expectation value $(\mathrm{VEV})$ of $\Sigma^{a}{ }_{b},\left\langle\Sigma_{b}^{a}\right\rangle=\operatorname{diag}\left(\frac{1}{3}, \frac{1}{3}, \frac{1}{3},-\frac{1}{2},-\frac{1}{2}\right) v_{G}$ breaks the $\mathrm{SU}(5)$ symmetry to $\mathrm{SU}(3)_{\mathrm{C}} \times \mathrm{SU}(2)_{\mathrm{L}} \times \mathrm{U}(1)_{\mathrm{Y}}$ at $\mu_{G} . \Delta \mathcal{W}_{\mathrm{SU}(5) \nu_{R}}$ is a dimension five operator, which is introduced in order to reproduce the realistic mass relations between the down-type quarks and charged leptons, as explained in Ref. [26]. 
The supermultiplets with the masses of order of the GUT scale such as $H_{C}$ and $\bar{H}_{C}$ are integrated out at $\mu_{G}$ and the effective theory below $\mu_{G}$ is the MSSM with the righthanded neutrinos described by the superpotential $\mathcal{W}_{\mathrm{MSSM} \nu_{R}}$ in Eq. (6) . The Yukawa coupling matrices in Eq. (6) are related to those in Eq. (15) as $\left(y_{U}\right)^{i j}=\left(\lambda_{U}\right)^{i j}$ and $\left(y_{N}\right)^{i j}=\left(\lambda_{N}\right)^{i j}$. $\left(\lambda_{D}\right)^{i j}$ is determined from $\left(y_{D}\right)^{i j}$ and $\left(y_{E}\right)^{i j}$, taking $O\left(\mu_{G} / \mu_{P}\right)$ corrections from $\Delta \mathcal{W}_{\mathrm{SU}(5) \nu_{R}}$ into account [26].

There are additional degrees of freedom in the matching relations between $y_{U, D, E, N}$ and $\lambda_{U, D, N}$, which cannot be determined from the quark and lepton masses and the CKM and PMNS matrices at low energy [22, 23, 38]. In the present analysis, we introduce only two relative phase parameters for simplicity, which corresponds to $\widehat{\Theta}_{L}$ in Ref. [23].

The SU(5) invariant and renormalizable soft breaking terms are written as

$$
\begin{aligned}
-\mathcal{L}_{\mathrm{soft}}^{\mathrm{SU}(5)}= & \frac{1}{2}\left(m_{T}^{2}\right)^{i j}\left(\tilde{T}_{i}^{*}\right)_{a b}\left(\tilde{T}_{j}\right)^{a b}+\left(m_{\bar{F}}^{2}\right)^{i j}\left(\tilde{\bar{F}}_{i}^{*}\right)^{a}\left(\tilde{\bar{F}}_{j}\right)_{a}+\left(m_{\bar{N}}^{2}\right)^{i j} \tilde{\bar{N}}_{i}^{*} \tilde{\bar{N}}_{j} \\
+ & \left(m_{H}^{2}\right) H^{*} H^{a}+\left(m_{\bar{H}}^{2}\right) \bar{H}^{* a} \bar{H}_{a}+\left(\frac{3}{2} B_{H} \lambda_{H} v_{G} \bar{H}_{a} H^{a}+\text { H.c. }\right)+\left(\text { terms with } \Sigma_{b}^{a}\right) \\
+ & \left\{\frac{1}{8} \epsilon_{a b c d e}\left(\tilde{\lambda}_{U}\right)^{i j}\left(\tilde{T}_{i}\right)^{a b}\left(\tilde{T}_{j}\right)^{c d} H^{e}+\left(\tilde{\lambda}_{D}\right)^{i j}\left(\tilde{\bar{F}}_{i}\right)_{a}\left(\tilde{T}_{j}\right)^{a b} \bar{H}_{b}\right. \\
& \left.+\left(\tilde{\lambda}_{N}\right)^{i j} \tilde{\bar{N}}_{i}\left(\tilde{\bar{F}}_{j}\right)_{a} H^{a}+\frac{1}{2}\left(\tilde{M}_{N}\right)^{i j} \tilde{\bar{N}}_{i} \tilde{\bar{N}}_{j}+\text { H.c. }\right\} \\
+ & \frac{1}{2} M_{5} \overline{\tilde{G}}_{5} \tilde{G}_{5},
\end{aligned}
$$

where $\tilde{T}_{i}, \tilde{\bar{F}}_{i}$, and $\tilde{\bar{N}}_{i}$ are the scalar components of $T_{i}, \bar{F}_{i}$, and $\bar{N}_{i}$, respectively; $H$ and $\bar{H}$ stand for the corresponding scalar components of the superfields denoted by the same symbols; and $\tilde{G}_{5}$ represents the $\mathrm{SU}(5)$ gaugino. We assume that the soft breaking terms are generated in a universal fashion at the Planck scale, $\mu_{P}$, i.e.

$$
\begin{aligned}
\left(m_{T}^{2}\right)^{i j} & =\left(m_{\bar{F}}^{2}\right)^{i j}=\left(m_{\bar{N}}^{2}\right)^{i j}=m_{0}^{2} \delta^{i j}, \\
(\widetilde{\lambda})^{i j} & =m_{0} A_{0}(\lambda)^{i j}, \quad\left(\lambda=\lambda_{U}, \lambda_{D}, \lambda_{N}\right), \\
M_{5} & =m_{1 / 2} .
\end{aligned}
$$

We solve the RG equations of the SU(5) SUSY GUT from $\mu_{P}$ to $\mu_{G}$ with Eq. (17) as boundary conditions at $\mu_{P}$, then those of MSSM with right-handed neutrinos between the $\mu_{G}$ and $\mu_{R}$. Finally, the squark and slepton mass matrices are obtained by the RG equations of the MSSM below $\mu_{R}$. 
Unlike the previous two models, a large flavor mixing in the neutrino sector can affect the right-handed down type squark sector because the lepton doublets and the down quarks are embedded in the same representation of $\mathrm{SU}(5)$. For a similar reason, the CKM mixing in the quark sector contributes to the mixing in the right-handed charged slepton sector. Quark flavor signals in models with a grand unification have been studied in literature [22, 23, 24]. It is shown in these papers that large contributions to $\epsilon_{K}$ and the $\mu \rightarrow e \gamma$ decay can arise from the new source of flavor mixing in the neutrino sector.

We study the same patterns of neutrino Yukawa couplings as those in MSSM with righthanded neutrinos, i.e. degenerate, non-degenerate (I) and non-degenerate (II) cases.

\section{A model with U(2) flavor symmetry}

There is a class of models which are intended to solve the flavor problem of the MSSM by introducing appropriate symmetry structure. U(2) flavor symmetry [39, 40] is a typical example of such models. We consider the model given in Ref. [40]. In this model, the quark and lepton supermultiplets in the first and the second generations transform as doublets under the $\mathrm{U}(2)$ flavor symmetry, and the third generation and the Higgs supermultiplets are singlets under the $\mathrm{U}(2)$. In addition to the ordinary matter fields, we introduce the following superfields: a doublet $\Phi^{i}(-1)$, a symmetric tensor $S^{i j}(-2)$, and an antisymmetric tensor $A^{i j}(-2)$, where $i$ and $j$ run over the first two generations and the numbers in the parentheses represent the charge of the $\mathrm{U}(1)$ subgroup.

The $\mathrm{U}(2)$ invariant superpotential relevant to the quark Yukawa couplings is given as follows:

$$
\begin{aligned}
\mathcal{W}_{\mathrm{U}(2)}= & Y_{U}\left(\bar{U}_{3} Q_{3} H_{2}+\frac{b_{U}}{M_{F}} \Phi^{i} \bar{U}_{i} Q_{3} H_{2}+\frac{c_{U}}{M_{F}} \bar{U}_{3} \Phi^{i} Q_{i} H_{2}\right. \\
& \left.+\frac{d_{U}}{M_{F}} S^{i j} \bar{U}_{i} Q_{j} H_{2}+\frac{a_{U}}{M_{F}} A^{i j} \bar{U}_{i} Q_{j} H_{2}\right) \\
& +Y_{D}\left(\bar{D}_{3} Q_{3} H_{1}+\frac{b_{D}}{M_{F}} \Phi^{i} \bar{D}_{i} Q_{3} H_{1}+\frac{c_{D}}{M_{F}} \bar{D}_{3} \Phi^{i} Q_{i} H_{1}\right. \\
& \left.+\frac{d_{D}}{M_{F}} S^{i j} \bar{D}_{i} Q_{j} H_{1}+\frac{a_{D}}{M_{F}} A^{i j} \bar{D}_{i} Q_{j} H_{1}\right),
\end{aligned}
$$

where $M_{F}$ is the scale of the flavor symmetry, and $Y_{Q}, a_{Q}, b_{Q}, c_{Q}$, and $d_{Q}(Q=U, D)$ are dimensionless coupling constants. Dimension five and higher dimensional operators are 
neglected in the superpotential in Eq. (18). Absolute values of the above dimensionless coupling constants except for $Y_{D}$ are supposed to be of $O(1)$.

The breaking pattern of the $\mathrm{U}(2)$ symmetry is assumed to be

$$
\mathrm{U}(2) \rightarrow \mathrm{U}(1) \rightarrow \text { no symmetry }
$$

in order to reproduce the preferable quark Yukawa coupling matrices which can explain the mass eigenvalues and the mixing of quarks. The first breaking is induced by VEV's of $\Phi^{i}$ and $S^{i j}$, and the second one by a VEV of $A^{i j}$. These VEV's are given as

$$
\frac{\left\langle\Phi^{i}\right\rangle}{M_{F}}=\delta^{i 2} \epsilon, \frac{\left\langle S^{i j}\right\rangle}{M_{F}}=\delta^{i 2} \delta^{j 2} \epsilon, \frac{\left\langle A^{i j}\right\rangle}{M_{F}}=\epsilon^{i j} \epsilon^{\prime},
$$

where $\epsilon$ and $\epsilon^{\prime}$ are taken to be real without loss of generality. Because $\epsilon$ and $\epsilon^{\prime}$ are order parameters of the $\mathrm{U}(2)$ and $\mathrm{U}(1)$ symmetry breaking respectively, they satisfy $\epsilon^{\prime} \ll \epsilon$. Note that $\left\langle S^{i j}\right\rangle$ is chosen to leave the U(1) unbroken. With the breaking pattern given in Eq. (19), we obtain the quark Yukawa coupling matrix $y_{Q}$ as

$$
y_{Q}^{i j}=Y_{Q}\left(\begin{array}{ccc}
0 & a_{Q} \epsilon^{\prime} & 0 \\
-a_{Q} \epsilon^{\prime} & d_{Q} \epsilon & b_{Q} \epsilon \\
0 & c_{Q} \epsilon & 1
\end{array}\right), \quad Q=U, D .
$$

The $\mathrm{U}(2)$ symmetry controls not only the superpotential but also the soft breaking terms. After the U(2) broken with the pattern in Eq. (19), the squark mass matrices $m_{X}^{2}$ can be obtained as

$$
m_{X}^{2}=\left(m_{0}^{X}\right)^{2}\left(\begin{array}{ccc}
1 & 0 & 0 \\
0 & 1+r_{22}^{X} \epsilon^{2} & r_{23}^{X} \epsilon \\
0 & r_{23}^{X *} \epsilon & r_{33}^{X}
\end{array}\right), X=Q, U, D
$$

where $r_{i j}^{X}$ are dimensionless parameters of $\mathcal{O}(1)$. As for the squark $A$ terms, they have the same structure as the quark Yukawa coupling matrices:

$$
A_{Q}^{i j}=A_{Q}^{0} Y_{Q}\left(\begin{array}{ccc}
0 & \tilde{a}_{Q} \epsilon^{\prime} & 0 \\
-\tilde{a}_{Q} \epsilon^{\prime} & \tilde{d}_{Q} \epsilon & \tilde{b}_{Q} \epsilon \\
0 & \tilde{c}_{Q} \epsilon & 1
\end{array}\right), \quad Q=U, D .
$$

In general, though being of $\mathcal{O}(1), \tilde{a}_{Q}, \tilde{b}_{Q}, \tilde{c}_{Q}$, and $\tilde{d}_{Q}$ take different values from the corresponding parameters in Eq. (21), and we expect no exact universality of the $A$ terms in this model. 
With the help of the $\mathrm{U}(2)$ symmetry, the masses of the first and second generation squarks are naturally degenerate. On the other hand, the mass of the third generation squarks may be separated from the others. There exist flavor mixings of $\mathcal{O}(\epsilon)$ between the second and the third generations of squarks. These are new sources of flavor mixing besides the CKM matrix.

There are several efforts to explain the observed neutrino masses and mixings in SUSY models with the $\mathrm{U}(2)$ flavor symmetry (or its discrete relatives) [41]. Unlike the quark sector, application of the $\mathrm{U}(2)$ symmetry to the lepton sector is not straightforward because of the large mixings of the neutrinos. Therefore we focus on the quark sector in the following analysis, taking the same boundary conditions as Eq. (4) for the slepton sector.

\section{B. Treatments of radiative breaking of the electroweak symmetry}

In the models we consider, SUSY parameters such as $m_{0}, m_{1 / 2}, A_{0}$ etc. are given at the high energy cut-off scale. In order to analyze flavor signals, we need to connect the parameters at the cut-off scale and those at the electroweak scale with help of the renormalization group equations. In the present work, we adopt the following procedure to determine the parameters at the electroweak scale.

1. The masses of quarks and leptons and the mixings (the CKM and PMNS matrices) are given as inputs at the electroweak scale, $\mu_{W}=M_{Z}$. These masses are running masses in the standard model. The Yukawa couplings and the coupling matrix of the dimension five operator $K_{\nu}$ in Eq. (6) are determined by these masses and another input parameter $\tan \beta \equiv\left\langle h_{2}\right\rangle /\left\langle h_{1}\right\rangle$.

2. Two-loop RGEs for the Yukawa couplings and $K_{\nu}$, as well as the gauge coupling constants, are solved up to a high energy cut-off scale with the boundary conditions given at $\mu_{W}$. The cut-off scale is taken as the GUT scale, $\mu_{G}$, for the mSUGRA, the MSSM with right-handed neutrinos, and the $\mathrm{U}(2)$ model and the Planck scale, $\mu_{P}$, for the SU(5) SUSY GUT with right-handed neutrinos. By this procedure, we calculate the parameters in the superpotential at the cut-off scale. A schematic picture of the

cut-off scale involved in these models is displayed in Fig. 1. Here, the $\overline{\mathrm{DR}}^{\prime}$ scheme [42] is adopted as a renormalization scheme. 
In the MSSM with right-handed neutrinos and SU(5) SUSY GUT with right-handed neutrinos, we decompose $K_{\nu}$ to $y_{N}$ and $M_{N}$ at the $\mu_{R}$ scale so that they satisfy the seesaw relation, Eq. (6). In the SU(5) SUSY GUT with right-handed neutrinos the parameters in $\mathcal{W}_{\mathrm{SU}(5) \nu_{R}}$ are matched with the MSSM with right-handed neutrinos at the GUT scale, $\mu_{G}$.

3. The boundary conditions for the soft SUSY breaking parameters are set at the cutoff scale as Eq. (4) for the mSUGRA, Eq. (44) and (10) for the MSSM with righthanded neutrinos, Eq. (17) for the SU(5) SUSY GUT with right-handed neutrinos, and Eq. (22) for the U(2) model. We take the same boundary conditions for the $A$ parameters in the $\mathrm{U}(2)$ model as the mSUGRA case for simplicity.

4. With help of two-loop RGEs, we evaluate the soft breaking terms at a typical SUSY breaking scale, $\mu_{S}=1 \mathrm{TeV}$, and calculate the SUSY masses and mixings at the leading order which are considered as $\overline{\mathrm{DR}}^{\prime}$ masses. For the masses of the Higgs bosons, the one loop corrections are included.

Then we set the value of $\mu$ and $B$ so that the tadpole diagrams of the Higgs bosons up to one loop level vanish.

Then running the $\mu$ to the electroweak scale $M_{Z}$, we obtain the $\mu$ at $M_{Z}$.

5. The SUSY threshold corrections to the gauge couplings and the masses of quarks and leptons are evaluated in order to determine $\overline{\mathrm{DR}}^{\prime}$ gauge couplings, $\overline{\mathrm{DR}}^{\prime}$ Higgs vev, and $\overline{\mathrm{DR}}^{\prime}$ masses of the matter fermions in the MSSM which lead to the $\overline{\mathrm{DR}}^{\prime}$ Yukawa couplings, according to Ref. [43].

6. We iterate from 2 to 5 in the above list until the numerical behavior converges.

7. The physical mass spectrum of SUSY particles is calculated at the $M_{Z}$ scale up to one loop level [43]. The flavor observables are also calculated with the parameters determined at the $M_{Z}$ scale.

In comparison with the previous work, two-loop RGEs for the running of SUSY parameters are used and the one loop SUSY threshold corrections at the electroweak scale are included in the calculation of this work. 
mSUGRA

MSSM with RN

SU(5) GUT with RN

$\mathrm{U}(2)$ model

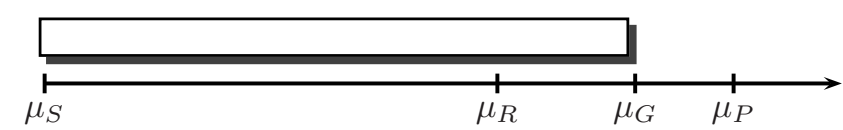

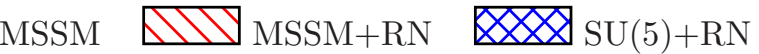

FIG. 1: The cut-off scales and the models. RN stands for right-handed neutrinos.

\section{NUMERICAL ANALYSIS}

\section{A. Flavor observables}

The flavor observables considered in the following are the $K^{0}-\bar{K}^{0}, B_{d}-\bar{B}_{d}$ and $B_{s}-\bar{B}_{s}$ mixings, both the direct and mixing induced $\mathrm{CP}$ asymmetries of $b \rightarrow s \gamma$ and $b \rightarrow d \gamma$, and the time dependent $\mathrm{CP}$ asymmetry of $B \rightarrow \phi K_{S}$. The branching ratios of the lepton flavor violating decay processes $\mu \rightarrow e \gamma, \tau \rightarrow \mu \gamma$ and $\tau \rightarrow e \gamma$ are also evaluated in the MSSM with right-handed neutrinos and SU(5) SUSY GUT with right-handed neutrinos. As mentioned in Sec. IIA4, we do not consider the flavor signals in the lepton sector for the $\mathrm{U}(2)$ model. Here we show the calculation methods of the flavor observables briefly. Detail on the calculation methods of the flavor observables is available in Ref. [22, 26, 27].

1. $K-\bar{K}$ and $B-\bar{B}$ mixings

The $K^{0}-\bar{K}^{0}, B_{d}-\bar{B}_{d}$ and $B_{s}-\bar{B}_{s}$ mixings are described by the effective Lagrangian of the following form:

$$
\begin{aligned}
\mathcal{L}_{\Delta F=2}= & C_{L L}\left(\bar{q}_{L}^{\alpha} \gamma^{\mu} Q_{L \alpha}\right)\left(\bar{q}_{L}^{\beta} \gamma_{\mu} Q_{L \beta}\right)+C_{R R}\left(\bar{q}_{R}^{\alpha} \gamma^{\mu} Q_{R \alpha}\right)\left(\bar{q}_{R}^{\beta} \gamma_{\mu} Q_{R \beta}\right) \\
& +C_{L R}^{(1)}\left(\bar{q}_{R}^{\alpha} Q_{L \alpha}\right)\left(\bar{q}_{L}^{\beta} Q_{R \beta}\right)+C_{L R}^{(2)}\left(\bar{q}_{R}^{\alpha} Q_{L \beta}\right)\left(\bar{q}_{L}^{\beta} Q_{R \alpha}\right) \\
& +\tilde{C}_{L L}^{(1)}\left(\bar{q}_{R}^{\alpha} Q_{L \alpha}\right)\left(\bar{q}_{R}^{\beta} Q_{L \beta}\right)+\tilde{C}_{L L}^{(2)}\left(\bar{q}_{R}^{\alpha} Q_{L \beta}\right)\left(\bar{q}_{R}^{\beta} Q_{L \alpha}\right) \\
& +\tilde{C}_{R R}^{(1)}\left(\bar{q}_{L}^{\alpha} Q_{R \alpha}\right)\left(\bar{q}_{L}^{\beta} Q_{R \beta}\right)+\tilde{C}_{R R}^{(2)}\left(\bar{q}_{L}^{\alpha} Q_{R \beta}\right)\left(\bar{q}_{L}^{\beta} Q_{R \alpha}\right),
\end{aligned}
$$

where $(q, Q)=(d, b),(s, b)$ and $(d, s)$ for the $B_{d}-\bar{B}_{d}, B_{s}-\bar{B}_{s}$ and $K^{0}-\bar{K}^{0}$ mixings, respectively, and the suffices $\alpha$ and $\beta$ denote color indices. New physics contributions to 


\begin{tabular}{cccccccc}
\hline$P$ & $f_{P}(\mathrm{MeV})$ & $B_{P}$ & $B_{P}^{L R(1)}$ & $B_{P}^{L R(2)}$ & $\tilde{B}_{P}^{R R(1)}$ & $\tilde{B}_{P}^{R R(2)}$ \\
\hline$K$ & 159.8 & 0.63 & 1.03 & 0.77 & 0.59 & 0.85 \\
$B_{d}$ & 198 & 0.87 & 1.15 & 1.72 & 0.79 & 0.92 \\
$B_{s}$ & 239 & 0.87 & 1.16 & 1.75 & 0.80 & 0.94 \\
\hline
\end{tabular}

TABLE I: Decay constants and bag parameters for the $B^{0}-\bar{B}^{0}$ and the $K^{0}-\bar{K}^{0}$ mixing matrix elements [44] used in the numerical calculation. Here $f_{K}$ is the experimental value.

the Wilson coefficients C's, as well as the SM ones, are obtained by calculating relevant box diagrams. Explicit formulae of the coefficients are found in e.g. Ref. [22]. The mixing matrix elements $M_{12}\left(B_{d}\right), M_{12}\left(B_{s}\right)$, and $M_{12}(K)$ are given as

$$
M_{12}(P)=-\frac{1}{2 m_{P}}\left\langle P\left|\mathcal{L}_{\Delta F=2}\right| \bar{P}\right\rangle
$$

where $P=B_{d}, B_{s}, K^{0}$.

In the evaluation of the matrix elements $\left\langle P\left|\mathcal{L}_{\Delta F=2}\right| \bar{P}\right\rangle$, we parameterize the matrix elements of the operators in Eq. (24) as

$$
\begin{aligned}
\left\langle K^{0}\left|\left(\bar{d}_{L}^{\alpha} \gamma^{\mu} s_{L \alpha}\right)\left(\bar{d}_{L}^{\beta} \gamma_{\mu} s_{L \beta}\right)\right| \bar{K}^{0}\right\rangle & =\frac{2}{3} m_{K}^{2} f_{K}^{2} B_{K} \\
\left\langle K^{0}\left|\left(\bar{d}_{R}^{\alpha} s_{L \alpha}\right)\left(\bar{d}_{L}^{\beta} s_{R \beta}\right)\right| \bar{K}^{0}\right\rangle & =\frac{1}{2}\left(\frac{m_{K}}{m_{s}+m_{d}}\right)^{2} m_{K}^{2} f_{K}^{2} B_{K}^{L R(1)} \\
\left\langle K^{0}\left|\left(\bar{d}_{R}^{\alpha} s_{L \beta}\right)\left(\bar{d}_{L}^{\beta} s_{R \alpha}\right)\right| \bar{K}^{0}\right\rangle & =\frac{1}{6}\left(\frac{m_{K}}{m_{s}+m_{d}}\right)^{2} m_{K}^{2} f_{K}^{2} B_{K}^{L R(2)} \\
\left\langle K^{0}\left|\left(\bar{d}_{L}^{\alpha} s_{R \alpha}\right)\left(\bar{d}_{L}^{\beta} s_{R \beta}\right)\right| \bar{K}^{0}\right\rangle & =-\frac{5}{12}\left(\frac{m_{K}}{m_{s}+m_{d}}\right)^{2} m_{K}^{2} f_{K}^{2} \tilde{B}_{K}^{R R(1)} \\
\left\langle K^{0}\left|\left(\bar{d}_{L}^{\alpha} s_{R \beta}\right)\left(\bar{d}_{L}^{\beta} s_{R \alpha}\right)\right| \bar{K}^{0}\right\rangle & =\frac{1}{12}\left(\frac{m_{K}}{m_{s}+m_{d}}\right)^{2} m_{K}^{2} f_{K}^{2} \tilde{B}_{K}^{R R(2)}
\end{aligned}
$$

where $B_{K}, B_{K}^{L R(1,2)}$, and $\tilde{B}_{K}^{R R(1,2)}$ are bag parameters. $B-\bar{B}$ mixing matrix elements are also defined in the same way. The bag parameters of $B$ and $K$ mesons and the decay constants of the $B$ mesons are evaluated by the lattice QCD method [44]. We list the numerical values used in our calculation in Table I. 
The observables $\varepsilon_{K}, \Delta m_{B_{d}}$ and $\Delta m_{B_{s}}$ are expressed in terms of $M_{12}$ as

$$
\begin{aligned}
\varepsilon_{K} & =\frac{e^{i \pi / 4} \operatorname{Im} M_{12}(K)}{\sqrt{2} \Delta m_{K}}, \\
\Delta m_{B_{d}} & =2\left|M_{12}\left(B_{d}\right)\right|, \\
\Delta m_{B_{s}} & =2\left|M_{12}\left(B_{s}\right)\right| .
\end{aligned}
$$

\section{2. $C P$ asymmetries in $B$ meson decays}

The time-dependent CP asymmetry in the $B_{d}$ decays to a $\mathrm{CP}$ eigenstate $f_{\mathrm{CP}}$ is given by

$$
\begin{aligned}
\frac{\Gamma\left(\bar{B}_{d}(t) \rightarrow f_{\mathrm{CP}}\right)-\Gamma\left(B_{d}(t) \rightarrow f_{\mathrm{CP}}\right)}{\Gamma\left(\bar{B}_{d}(t) \rightarrow f_{\mathrm{CP}}\right)+\Gamma\left(B_{d}(t) \rightarrow f_{\mathrm{CP}}\right)}= & A_{\mathrm{CP}}\left(B_{d} \rightarrow f_{\mathrm{CP}}\right) \cos \Delta m_{B_{d}} t \\
& +S_{\mathrm{CP}}\left(B_{d} \rightarrow f_{\mathrm{CP}}\right) \sin \Delta m_{B_{d}} t
\end{aligned}
$$

where $A_{\mathrm{CP}}$ and $S_{\mathrm{CP}}$ are direct and indirect (mixing-induced) CP violation parameters, respectively.

For $f_{\mathrm{CP}}=J / \psi K_{S}$, the $b \rightarrow c \bar{c} s$ decay amplitude is assumed to be dominated by the tree level Standard Model contribution. Consequently, the direct CP asymmetry $A_{\mathrm{CP}}\left(B_{d} \rightarrow\right.$ $\left.J / \psi K_{S}\right)$ is negligibly small. The weak phase of the $b \rightarrow c \bar{c} s$ decay amplitude comes from a product of the CKM matrix elements $V_{c b} V_{c s}^{*}$, which is almost real by convention. Therefore we can write

$$
\begin{gathered}
\frac{\Gamma\left(\bar{B}_{d}(t) \rightarrow J / \psi K_{S}\right)-\Gamma\left(B_{d}(t) \rightarrow J / \psi K_{S}\right)}{\Gamma\left(\bar{B}_{d}(t) \rightarrow J / \psi K_{S}\right)+\Gamma\left(B_{d}(t) \rightarrow J / \psi K_{S}\right)}=S_{\mathrm{CP}}\left(B_{d} \rightarrow J / \psi K_{S}\right) \sin \Delta m_{B_{d}} t \\
S_{\mathrm{CP}}\left(B_{d} \rightarrow J / \psi K_{S}\right)=\sin \phi_{M}
\end{gathered}
$$

with $\phi_{M}$ being $e^{i \phi_{M}}=M_{12}\left(B_{d}\right) /\left|M_{12}\left(B_{d}\right)\right|$. In the Standard Model, $\phi_{M}=2 \phi_{1}=$ $2 \arg \left(-V_{c b}^{*} V_{c d} /\left(V_{t b}^{*} V_{t d}\right)\right)$. Experimentally, $\sin \phi_{M}$ can be determined by combining decay modes with the $b \rightarrow c \bar{c} s$ transition such as $B_{d} \rightarrow J / \psi K_{S}, B_{d} \rightarrow J / \psi K_{L}$, and $B_{d} \rightarrow \psi^{\prime} K_{S}$.

The time-dependent CP asymmetry in the $B_{s}$ decay is formulated in the same way. $B_{s} \rightarrow$ $J / \psi \phi$ is the $b \rightarrow c \bar{c} s$ mode of the $B_{s}$ decay, which corresponds to $B_{d} \rightarrow J / \psi K_{S}$. The mixinginduced CP violation parameter $S_{\mathrm{CP}}\left(B_{s} \rightarrow J / \psi \phi\right)$ is written as $S_{\mathrm{CP}}\left(B_{s} \rightarrow J / \psi \phi\right)=\sin \phi_{M_{s}}$ where $\phi_{M_{s}}$ is defined as $e^{i \phi_{M_{s}}}=M_{12}\left(B_{s}\right) /\left|M_{12}\left(B_{s}\right)\right|$. In actual extraction, the angular analysis is needed to separate CP odd and even contribution [12, 45]. The Standard Model prediction is given as $\left.\sin \phi_{M_{s}}\right|_{\mathrm{SM}} \simeq-0.04$. 
We also consider the decay mode $B_{d} \rightarrow \phi K_{S}$, which is supposed to be a pure $b \rightarrow s \bar{s} s$ process. The mixing-induced $\mathrm{CP}$ asymmetry $S_{\mathrm{CP}}\left(B_{d} \rightarrow \phi K_{S}\right)$, is given as

$$
S_{\mathrm{CP}}\left(B_{d} \rightarrow \phi K_{S}\right)=\frac{2 \operatorname{Im}\left(e^{-i \phi_{M}} \overline{\mathcal{A}} \mathcal{A}\right)}{|\mathcal{A}|^{2}+|\overline{\mathcal{A}}|^{2}},
$$

where $\mathcal{A}$ and $\overline{\mathcal{A}}$ denote decay amplitudes of $B_{d} \rightarrow \phi K$ and $\bar{B}_{d} \rightarrow \phi \bar{K}$ respectively. This quantity is expected to coincide with $S_{\mathrm{CP}}\left(B_{d} \rightarrow J / \psi K_{S}\right)$ within the SM. If there is sizable deviation, this will be an evidence of new physics beyond the SM in $b \rightarrow s$ transition. The calculation of the decay amplitude involves sizable uncertainty. Here we use a method based on the naive factorization. Details of the calculation of $\mathcal{A}$ are given in Refs. [23, 46].

As for the $b \rightarrow q \gamma(q=s, d)$ decays, both direct and mixing-induced CP asymmetries are considered, as well as the branching ratio $\mathrm{B}(b \rightarrow s \gamma)$ which provides a significant constraint on the parameter space. Relevant effective Lagrangian is given as

$$
\mathcal{L}=C_{2 L} \mathcal{O}_{2 L}+C_{2 L}^{\prime} \mathcal{O}_{2 L}^{\prime}-C_{7 L} \mathcal{O}_{7 L}-C_{8 L} \mathcal{O}_{8 L}+(L \leftrightarrow R)+\mathcal{L}_{4 q}
$$

The operators $\mathcal{O}$ 's are

$$
\begin{aligned}
\mathcal{O}_{2 L} & =\left(\bar{q}_{\alpha} \gamma^{\mu} c_{L \alpha}\right)\left(\bar{c}_{\beta} \gamma^{\mu} b_{L \beta}\right), \\
\mathcal{O}_{2 L}^{\prime} & =\left(\bar{q}_{\alpha} \gamma^{\mu} u_{L \alpha}\right)\left(\bar{u}_{\beta} \gamma^{\mu} b_{L \beta}\right)-\left(\bar{q}_{\alpha} \gamma^{\mu} c_{L \alpha}\right)\left(\bar{c}_{\beta} \gamma^{\mu} b_{L \beta}\right), \\
\mathcal{O}_{7 L} & =\frac{e}{16 \pi^{2}} m_{b} \bar{q} \frac{i}{2}\left[\gamma^{\mu}, \gamma^{\nu}\right] b_{R} F_{\mu \nu}, \\
\mathcal{O}_{8 L} & =\frac{g_{3}}{16 \pi^{2}} m_{b} \bar{q}^{\alpha} \frac{i}{2}\left[\gamma^{\mu}, \gamma^{\nu}\right] T_{\alpha \beta}^{(a)} b_{R}^{\beta} G_{\mu \nu}^{(a)},
\end{aligned}
$$

where $q$ is $s$ or $d$ for $b \rightarrow s \gamma$ or $b \rightarrow d \gamma$ decays, respectively. $\mathcal{L}_{4 q}$ denotes the terms with fourquark operators induced by loop effects. The Wilson coefficients $C_{2 L}$ and $C_{2 L}^{\prime}$ are dominated by the contributions from the tree level $W$ boson exchange. Therefore, $C_{2 L}^{\prime}=\epsilon_{u} C_{2 L}$ is satisfied, where $\epsilon_{u}=-V_{u q}^{*} V_{u b} /\left(V_{t q}^{*} V_{t b}\right)$. The direct CP asymmetry in the inclusive decays $B \rightarrow X_{q} \gamma(q=s, d)$ is given as [47]

$$
\begin{aligned}
A_{\mathrm{CP}}^{\operatorname{dir}}\left(B \rightarrow X_{q} \gamma\right)= & \frac{\Gamma\left(\bar{B} \rightarrow X_{q} \gamma\right)-\Gamma\left(B \rightarrow X_{\bar{q}} \gamma\right)}{\Gamma\left(\bar{B} \rightarrow X_{q} \gamma\right)+\Gamma\left(B \rightarrow X_{\bar{q}} \gamma\right)} \\
= & -\frac{\alpha_{3}}{\pi\left(\left|C_{7 L}\right|^{2}+\left|C_{7 R}\right|^{2}\right)}\left[-\operatorname{Im} r_{2} \operatorname{Im}\left[\left(1-\epsilon_{u}\right) C_{2 L} C_{7 L}^{*}\right]+\frac{80}{81} \pi \operatorname{Im}\left(\epsilon_{u} C_{2 L} C_{7 L}^{*}\right)\right. \\
& +\frac{8}{9} \pi \operatorname{Im}\left(C_{8 L} C_{7 L}^{*}\right)-\operatorname{Im} f_{27} \operatorname{Im}\left[\left(1-\epsilon_{u}\right) C_{2 L} C_{7 L}^{*}\right] \\
& \left.+\frac{1}{3} \operatorname{Im} f_{27} \operatorname{Im}\left[\left(1-\epsilon_{u}\right) C_{2 L} C_{8 L}^{*}\right]+(L \leftrightarrow R)\right]
\end{aligned}
$$


where the functions $r_{2}$ and $f_{27}$ for $B \rightarrow X_{s} \gamma$ are found in Ref. [48]. The mixing-induced $\mathrm{CP}$ asymmetry is defined for an exclusive $B_{d} \rightarrow M_{q} \gamma$ decay. $M_{q}$ denotes a hadronic $\mathrm{CP}$ eigenstate which includes a strange or down quark such as $K^{*}($ for $q=s)$ and $\rho(q=d)$. $S_{\mathrm{CP}}\left(B_{d} \rightarrow M_{q} \gamma\right)$ is given as [49]

$$
S_{\mathrm{CP}}\left(B_{d} \rightarrow M_{q} \gamma\right)=\frac{2 \operatorname{Im}\left(e^{-i \phi_{M}} C_{7 L} C_{7 R}\right)}{\left|C_{7 L}\right|^{2}+\left|C_{7 R}\right|^{2}}
$$

\section{Lepton flavor violation}

The effective Lagrangian for the lepton flavor violating $l_{j} \rightarrow l_{i} \gamma$ decay is written as

$$
\mathcal{L}_{\mathrm{LFV}}=-\frac{e}{16 \pi^{2}} m_{l_{j}} \bar{l}_{i} \frac{i}{2}\left[\gamma^{\mu}, \gamma^{\nu}\right]\left(A_{L}^{i j} P_{R}+A_{R}^{i j} P_{L}\right) l_{j} F_{\mu \nu}, \quad(i \neq j)
$$

where $P_{R}=\left(1+\gamma_{5}\right) / 2$ and $P_{L}=\left(1-\gamma_{5}\right) / 2$. The decay width is given by

$$
\Gamma\left(l_{j} \rightarrow l_{i} \gamma\right)=\frac{\alpha}{64 \pi^{2}} m_{l_{j}}^{5}\left(\left|A_{L}^{i j}\right|^{2}+\left|A_{R}^{i j}\right|^{2}\right)
$$

\section{Electric dipole moments}

Electric dipole moment $d_{f}$ of a fermion $f$ is defined as the coefficient in the effective Lagrangian

$$
\mathcal{L}=\frac{i}{2} d_{f} \bar{f} \frac{i}{2}\left[\gamma^{\mu}, \gamma^{\nu}\right] \gamma_{5} f F_{\mu \nu}
$$

In addition, chromo-electric dipole moments of quarks and the three-gluon operator [50] are taken into account for hadronic EDMs. Relevant effective Lagrangian is written as

$$
\mathcal{L}=\frac{i}{2} d_{q}^{C} \bar{q} \frac{i}{2}\left[\gamma^{\mu}, \gamma^{\nu}\right] \gamma_{5} T^{(a)} q G_{\mu \nu}^{(a)}+\frac{d^{G}}{6} f^{(a)(b)(c)} \epsilon^{\mu \nu \lambda \rho} G_{\mu \sigma}^{(a)} G_{\nu}^{(b) \sigma} G_{\lambda \rho}^{(a)}
$$

We calculate $d_{f}$ for quarks and leptons and $d_{q}^{C}$ with all the one-loop SUSY contributions [29, 30] and two-loop contributions given in Ref. [31]. $d^{G}$ is calculated according to Ref. [30].

The neutron and the mercury EDMs, $d(n)$ and $d(\mathrm{Hg})$, respectively, are written as linear combinations of $d_{q}, d_{q}^{C}$ and $d^{G}$ :

$$
d(h)=\sum_{q=u, d, s}\left[c_{q}(h) d_{q}+c_{q}^{C}(h) d_{q}^{C}\right]+c^{G}(h) d^{G}, \quad h=n, \mathrm{Hg} .
$$

Values of the coefficients used in our calculation are given in Table II. 


\begin{tabular}{ccccccccc}
\hline$h$ & $c_{u}$ & $c_{d}$ & $c_{s}$ & $c_{u}^{C}$ & $c_{d}^{C}$ & $c_{s}^{C}$ & $c^{G}$ & Ref. \\
\hline$n(\mathrm{NDA})$ & $-\frac{1}{3}$ & $\frac{4}{3}$ & 0 & $-\frac{1}{3} \frac{e}{4 \pi}$ & $\frac{4}{3} \frac{e}{4 \pi}$ & 0 & $-\frac{e}{2 \sqrt{2}} f_{\pi}$ & {$[30]$} \\
$n(\mathrm{ChPT})$ & 0 & 0 & 0 & $1.6 e$ & $1.3 e$ & $0.26 e$ & 0 & {$[33]$} \\
$\mathrm{Hg}$ & 0 & 0 & 0 & $0.0087 e$ & $-0.0087 e$ & $4.4 \times 10^{-5} e$ & 0 & {$[33]$} \\
\hline
\end{tabular}

TABLE II: Hadronic factors used in the calculation of EDMs.

There are large uncertainties in the estimation of the hadronic EDMs. Here we use the value of the neutron EDM obtained by the formulae based on the naive dimensional analysis (NDA) [30]. On the other hand, it has been pointed out that an evaluation with use of the chiral perturbation theory (ChPT) may give a much larger value of the neutron EDM, due to the chromo-electric dipole moment of the strange quark [33]. We later discuss how the numerical results change if the latter is applied.

\section{B. Input parameters and experimental constraints}

As input parameters at the low energy, the mass eigenvalues and the flavor mixing matrices of the quarks and leptons are used.

The CKM matrix elements $V_{u s}, V_{c b}$, and $\left|V_{u b}\right|$ are determined by measurements of the processes which are supposed to be dominated by the SM tree level contributions. We adopt $V_{u s}=0.224$ and $V_{c b}=0.0416$ in the following calculations. As for the $\left|V_{u b}\right|$, because the uncertainty is relatively large, we vary $\left|V_{u b}\right|$ within a range $3.0<\left|V_{u b}\right| / 10^{-3}<4.7$. The CKM phase is not yet determined by tree level processes free from new physics contributions. Therefore we vary the CKM phase $\phi_{3} \equiv \arg \left(-V_{u b}^{*} V_{u d} / V_{c b}^{*} V_{c d}\right)$ within $0<\phi_{3}<180^{\circ}$.

In the models with neutrino masses, we need to specify the parameters in the neutrino sector in addition to the quark Yukawa coupling constants. As for the mass eigenvalues, only the squared mass differences are measured by experiments as $\left|m_{\nu_{3}}^{2}-m_{\nu_{2}}^{2}\right|=2.5 \times 10^{-3} \mathrm{eV}^{2}$ and $m_{\nu_{2}}^{2}-m_{\nu_{1}}^{2}=8.0 \times 10^{-5} \mathrm{eV}^{2}$, so that one can consider several patterns of the neutrino mass spectrum. In the present analysis, we assume the normal hierarchical neutrino masses 
$m_{\nu_{1}} \ll m_{\nu_{2}} \ll m_{\nu_{3}}$ and $m_{\nu_{1}}=0.003 \mathrm{eV}$. We take the PMNS mixing matrix as

$$
V_{\mathrm{PMNS}}=\left(\begin{array}{ccc}
c_{\odot} c_{13} & s_{\odot} c_{13} & s_{13} \\
-s_{\odot} c_{\mathrm{atm}}-c_{\odot} s_{\mathrm{atm}} s_{13} & c_{\odot} c_{\mathrm{atm}}-s_{\odot} s_{\mathrm{atm}} s_{13} & s_{\mathrm{atm}} c_{13} \\
s_{\odot} s_{\mathrm{atm}}-c_{\odot} c_{\mathrm{atm}} s_{13} & -c_{\odot} s_{\mathrm{atm}}-s_{\odot} c_{\mathrm{atm}} s_{13} & c_{\mathrm{atm}} c_{13}
\end{array}\right),
$$

$\left(c_{i}=\cos \theta_{i}, s_{i}=\sin \theta_{i}\right)$ with $\sin ^{2} 2 \theta_{\mathrm{atm}}=1, \tan ^{2} \theta_{\odot}=0.4$, and $\sin ^{2} 2 \theta_{13}=0$. These mixing angles are consistent with the observed solar and atmospheric neutrino oscillations [2], the K2K experiment [3], and the KamLAND experiment [4]. Only the upper bound of $\sin ^{2} 2 \theta_{13}$ is obtained by reactor experiments [5], and we take the above value as an illustration. We ignore the Dirac and Majorana CP phases in the neutrino sector for simplicity, though they can affect the analysis of the lepton flavor violations [51].

We have nine extra parameters to specify the neutrino Yukawa coupling and right-handed neutrino mass matrices. In the non-degenerate cases, we generally parametrize the neutrino Yukawa coupling matrix as

$$
\begin{aligned}
y_{N}= & \hat{y}_{N} V_{L}, \\
\hat{y}_{N}= & \operatorname{diag}\left(\hat{y}_{1}, \hat{y}_{2}, \hat{y}_{3}\right), \\
V_{L}= & \left(\begin{array}{ccc}
\bar{c}_{12} \bar{c}_{13} & \bar{s}_{12} \bar{c}_{13} & \bar{s}_{13} e^{-i \bar{\delta}_{13}} \\
-\bar{s}_{12} \bar{c}_{23}-\bar{c}_{12} \bar{s}_{23} \bar{s}_{13} e^{i \bar{\delta}_{13}} & \bar{c}_{12} \bar{c}_{23}-\bar{s}_{12} \bar{s}_{23} \bar{s}_{13} e^{i \bar{\delta}_{13}} & \bar{s}_{23} \bar{c}_{13} \\
\bar{s}_{12} \bar{s}_{23}-\bar{c}_{12} \bar{c}_{23} \bar{s}_{13} e^{i \bar{\delta}_{13}} & -\bar{c}_{12} \bar{s}_{23}-\bar{s}_{12} \bar{c}_{23} \bar{s}_{13} e^{i \bar{\delta}_{13}} & \bar{c}_{23} \bar{c}_{13}
\end{array}\right) \\
& \times \operatorname{diag}\left(e^{i \bar{\psi}_{13}}, e^{i \bar{\psi}_{23}}, 1\right) e^{-i\left(\bar{\psi}_{13}+\bar{\psi}_{23}\right) / 3},
\end{aligned}
$$

where $\bar{s}_{i j}=\sin \bar{\theta}_{i j}$ and $\bar{c}_{i j}=\cos \bar{\theta}_{i j}$. The right-handed neutrino mass matrix is written as

$$
M_{N}=\hat{y}_{N} V_{L} K_{\nu}^{-1} V_{L}^{T} \hat{y}_{N}
$$

We take the input parameters as shown in Table III, which provide us appropriate $y_{N}$ of the structure (13) and (14). After calculating $M_{N}$, we rescale $\hat{y}_{N}$ (and $M_{N}$ ) such that $M_{N}$ satisfies $\operatorname{det} M_{N}=\mu_{R}^{3}$.

As for the SUSY parameters, we take the convention that the unified gaugino mass $m_{1 / 2}$ is real. As already described in Sec. IIA 1, it is known that $\phi_{\mu}$ is strongly constrained by the upper bounds of EDMs, while the corresponding constraint on $\phi_{A}$ is not so tight [29, 30]. Thus we fix $\phi_{\mu}=0^{\circ}$ at the electroweak scale. We scan the SUSY breaking parameters within the ranges $0 \leq m_{0} \leq 4 \mathrm{TeV}, 0<m_{1 / 2} \leq 1 \mathrm{TeV},\left|A_{0}\right| \leq 4$ and $-180^{\circ}<\phi_{A} \leq 180^{\circ}$. 


\begin{tabular}{cccccccccc}
\hline Case & $\hat{y}_{1}$ & $\hat{y}_{2}$ & $\hat{y}_{3}$ & $\bar{s}_{12}$ & $\bar{s}_{23}$ & $\bar{s}_{13}$ & $\bar{\delta}_{13}$ & $\bar{\psi}_{13}$ & $\bar{\psi}_{23}$ \\
\hline (I) & 0.884 & 1.69 & 2.69 & 0 & -0.666 & 0 & 0 & 0 & 0 \\
(II) & 1.49 & 2.83 & 2.79 & 0 & 0 & 0.435 & 0 & 0 & 0 \\
\hline
\end{tabular}

TABLE III: Input parameters for the neutrino Yukawa coupling matrix in the non-degenerate cases.

In the SU(5) SUSY GUT with right-handed neutrinos, we also vary the two phase parameters, which are introduced at the GUT scale matching as mentioned in Sec. IIA3, within the whole range $\left\{-180^{\circ}, 180^{\circ}\right\}$.

In the $\mathrm{U}(2)$ model, the flavor symmetry breaking parameters $\epsilon$ and $\epsilon^{\prime}$ are fixed to be $\epsilon=0.04$ and $\epsilon^{\prime}=0.008$, and the parameters in the quark Yukawa coupling matrices are determined so that the CKM matrix and the quark masses are reproduced. There are six independent $O(1)$ parameters in the quark Yukawa coupling matrices of the form (21) for given quark masses and the CKM matrix. We scan those free parameters as inputs. For the squark mass matrices (22), we make an assumption

$$
m_{0}^{Q 2}=m_{0}^{U 2}=m_{0}^{D 2}=m_{0}^{2}
$$

and scan the range of $m_{0}$ as $0<m_{0}<4 \mathrm{TeV}$. Dimensionless parameters in Eq. (22) are varied within the ranges $0.4 \leq r_{22}^{X}, r_{33}^{X},\left|r_{23}^{X}\right| \leq 2.5$ and $-180^{\circ}<\arg r_{23}^{X} \leq 180^{\circ}$. We assume that the boundary conditions for the $A$ parameters are the same as the mSUGRA case for simplicity.

In order to constrain the parameter space, we consider the following experimental results:

- Lower limits on the masses of SUSY particles and the Higgs bosons given by direct searches in collider experiments [52].

- Branching ratio of the $b \rightarrow s \gamma$ decay: $\mathrm{B}(b \rightarrow s \gamma)=\left(3.55 \pm 0.24_{-0.10}^{+0.09} \pm 0.03\right) \times 10^{-4}$ [53]. We take the allowed range for the calculated branching ratio as $2.85 \times 10^{-4}<$ $\mathrm{B}(b \rightarrow s \gamma)<4.25 \times 10^{-4}$, taking also account of theoretical uncertainties.

- Upper bounds of the branching ratios of the $\mu \rightarrow e \gamma, \tau \rightarrow \mu \gamma$ and $\tau \rightarrow e \gamma$ decays for the MSSM with right-handed neutrinos and SUSY GUT cases: $\mathrm{B}(\mu \rightarrow e \gamma)<1.2 \times 10^{-11}$ [54], $\mathrm{B}(\tau \rightarrow \mu \gamma)<6.8 \times 10^{-8}[55]$ and $\mathrm{B}(\tau \rightarrow e \gamma)<1.1 \times 10^{-7}$. [56]

- Upper bounds of EDMs of ${ }^{199} \mathrm{Hg}$, the neutron and the electron: $\left|d_{\mathrm{Hg}}\right|<2.1 \times 10^{-28} e \cdot \mathrm{cm}$ [57], $\left|d_{n}\right|<2.9 \times 10^{-26} e \cdot \mathrm{cm}$ [58] and $\left|d_{e}\right|<1.6 \times 10^{-27} e \cdot \mathrm{cm}[59]$. 
- The CP violation parameter $\varepsilon_{K}$ in the $K^{0}-\bar{K}^{0}$ mixing $\left|\varepsilon_{K}\right|=(2.232 \pm 0.007) \times 10^{-3}$ and the $B_{d}-\bar{B}_{d}$ and the $B_{s}-\bar{B}_{s}$ mixing parameters $\Delta m_{B_{d}}=0.507 \pm 0.005 \mathrm{ps}^{-1}$ [60] and $\Delta m_{B_{s}}=17.77 \pm 0.10 \pm 0.07 \mathrm{ps}^{-1}[\underline{61}]$. Theoretical uncertainties in these quantities are larger than the experimental ones. For the $B-\bar{B}$ mixings, $1 \sigma$ uncertainties of the decay constants $f_{B_{d, s}}$ and of the bag parameters $B_{B_{d}, s}$ are evaluated as 10 percent and 8 percent, respectively [44]. In the present analysis, we calculate $\Delta m_{B_{d, s}}$ with a fixed set of hadronic parameters as listed in Table $\llbracket$ and allow \pm 40 percent deviations from the experimental central values. We expect that these ranges provide typical $2-3 \sigma$ allowed intervals. In addition, the ratio of the hadronic parameters $\xi \equiv f_{B_{s}} \sqrt{B_{B_{s}}} /\left(f_{B_{d}} \sqrt{B_{B_{s}}}\right)$ is evaluated with better accuracy. The uncertainty of $\xi$ is evaluated as \pm 4 percent [44]. Therefore we also require that the calculated ratio $\Delta m_{B_{s}} / \Delta m_{B_{d}}$, which is proportional to $\xi^{2}$, be within \pm 20 percent range of the central value. For $\varepsilon_{K}$ we assign \pm 15 percent uncertainty.

- CP asymmetry in the $B_{d} \rightarrow J / \psi K_{S}$ decay and related modes observed at the $B$ factory experiments: $\left.\sin 2 \phi_{1}\right|_{c \bar{c} s}=0.678 \pm 0.025$ [60]. We take the allowed range for the calculated value as $0.628<S_{\mathrm{CP}}\left(B_{d} \rightarrow J / \psi K_{S}\right)<0.728$, which is a simple $2 \sigma$ interval, since the theoretical uncertainty of this asymmetry is expected to be small.

\section{Numerical results}

\section{Allowed parameter region from the radiative electroweak symmetry breaking condition}

Before presenting flavor signals, we first discuss the SUSY parameter space which is allowed by the radiative electroweak symmetry breaking condition and experimental constraints.

In Fig. 2, we show the allowed region in the $m_{0}$ and $m_{1 / 2}$ plane for the mSUGRA, MSSM with right-handed neutrinos, and SU(5) SUSY GUT with right-handed neutrinos. Parameters other than $m_{0}$ and $m_{1 / 2}$ are fixed as indicated in each plot. Contours of $|\mu|$ determined from the electroweak symmetry breaking condition are also shown. In mSUGRA, the parameter region is mainly constrained by the lower limit on the chargino mass, the limit on the lightest Higgs boson mass, the branching ratio of $b \rightarrow s \gamma$ decay, and the requirement that the lightest supersymmetric particle (LSP) is neutral. When the neutrino Yukawa 


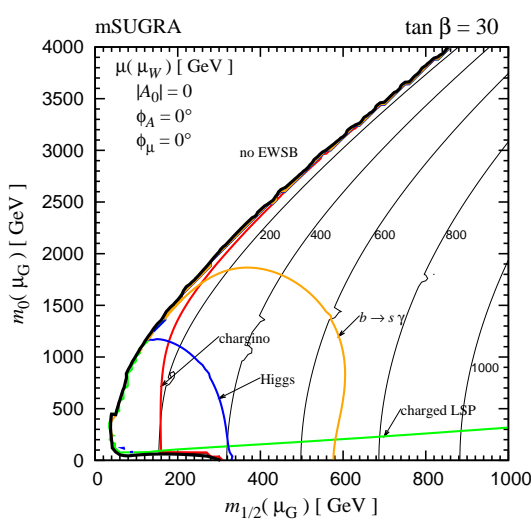

(a)

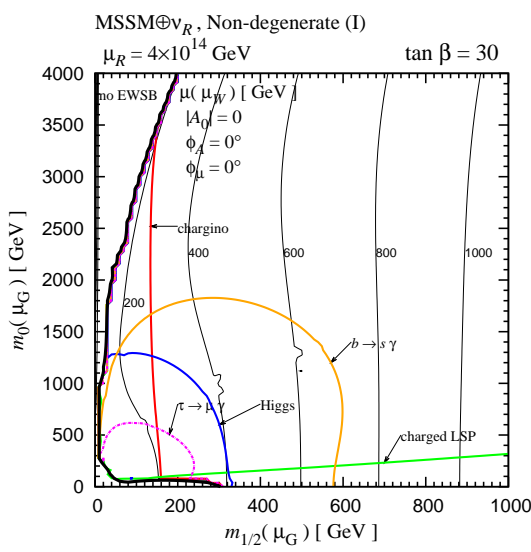

(d)

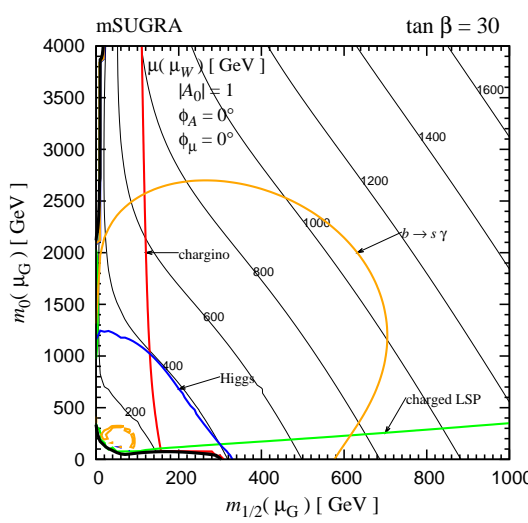

(b)

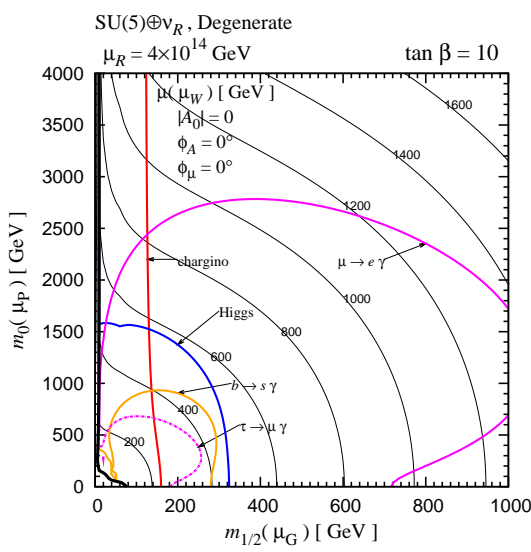

(e)

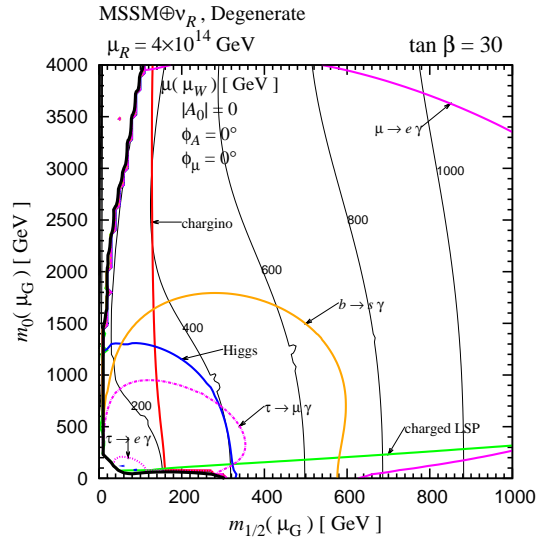

(c)

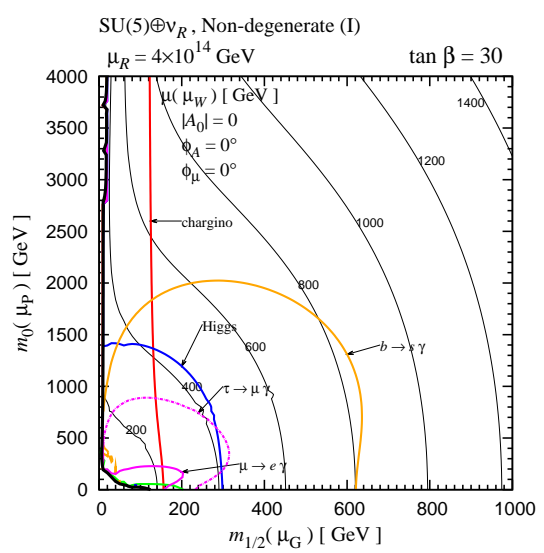

(f)

FIG. 2: (Color online) Contour plots of the value of $|\mu|$ on $m_{0}$ and $m_{1 / 2}$ plane for fixed $\tan \beta$ and $A_{0}$ in mSUGRA ((a) and (b)), MSSM with right-handed neutrinos ((c) and (d)) and SU(5) SUSY GUT with right-handed neutrinos models ((e) and (f)). Each thick black line shows the boundary of the region where correct electroweak symmetry breaking occurs. In the regions below the lines labeled with "charged LSP" (green) in (a)-(d), the LSP is a charged particle. Boundaries of excluded regions which come from the chargino mass (red), the Higgs boson mass (blue) and $\mathrm{B}(b \rightarrow s \gamma)$ (orange) are shown in each plot. Regions excluded by the lepton flavor violating processes are also shown in (c)-(f) (magenta).

couplings are relevant, the lepton flavor violating decays are enhanced. As a result, a large portion is excluded due to the experimental upper limit on the branching ratio of $\mu \rightarrow e \gamma$ for MSSM with right-handed neutrinos and SU(5) SUSY GUT with right-handed neutrinos. Notice that we take CP violating SUSY phases to be vanishing in these plots. A significant 
potion of the parameter space is excluded due to the experimental limits on EDMs if we take non-vanishing SUSY CP phases.

In the plot for mSUGRA with $\left|A_{0}\right|=0$ (Fig. $2(\mathrm{a})$ ), the $m_{0} \gg m_{1 / 2}$ region is excluded because the electroweak symmetry breaking cannot be satisfied, namely there is no solution with $|\mu|^{2} \geq 0$ for this region. The allowed region near the boundary corresponds to socalled "focus point" region [62] where the LSP is the lightest neutralino with significant higgsino component. This region is one of the favored regions in the context of the cosmic dark matter study [63]. The pair annihilation of the lightest neutralino into $W$ boson or $Z$ boson pair is enhanced by the gauge interaction of the higgsino component, so that the relic abundance of the LSP becomes suitable for the cold dark matter density. However, in $\left|A_{0}\right|=1$ case (Fig. 2(b)), such region disappears because the $A$-terms affect the running of the Higgs mass parameter $m_{H_{2}}^{2}$ so that a sufficiently large $|\mu|^{2}$ is realized. The "focus point" like region disappears also in the cases with right-handed neutrinos (Fig. 2(c)-(f)), since the large neutrino Yukawa coupling affects the running of $m_{H_{2}}^{2}$ in a similar way.

In the cases of mSUGRA and MSSM with right-handed neutrinos (Fig. 2(a)-(d)), the $m_{0} \ll m_{1 / 2}$ region is excluded because the LSP is the lightest charged slepton. The allowed region near the boundary provides another dark matter favored region [64]. The coannihilation effect among the LSP (neutralino) and the next-to-LSP (slepton), which are nearly degenerate in mass, provides an appropriate relic abundance of LSP. On the other hand, in the SU(5) SUSY GUT with right-handed neutrinos (Fig. 2(e), (f)), the running between the Planck and the GUT scales induces positive contribution to the slepton mass squared, which makes the charged slepton heavier than the lightest neutralino even in the $m_{0} \ll m_{1 / 2}$ region. Therefore "charged LSP" or "stau coannihilation" region disappears in the SU(5) SUSY GUT with right-handed neutrinos.

The disappearance of the "focus point" like region due to the effect of the neutrino Yukawa couplings and the disappearance of the "charged LSP" region caused by the running between the Planck and the GUT scales are previously observed in Ref. [25], where the SO(10) SUSY GUT is considered. 


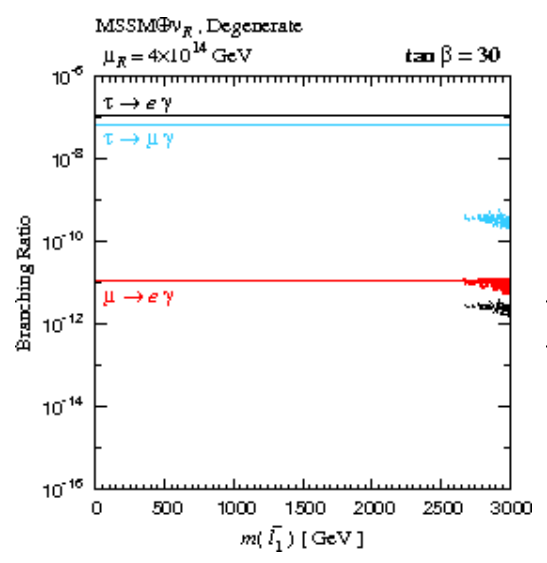

(a)

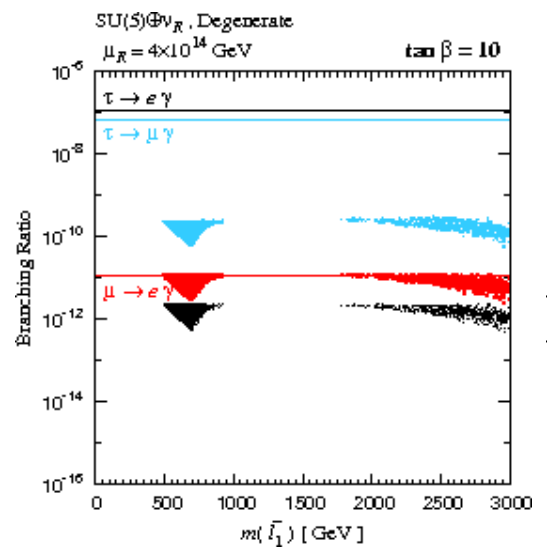

(d)

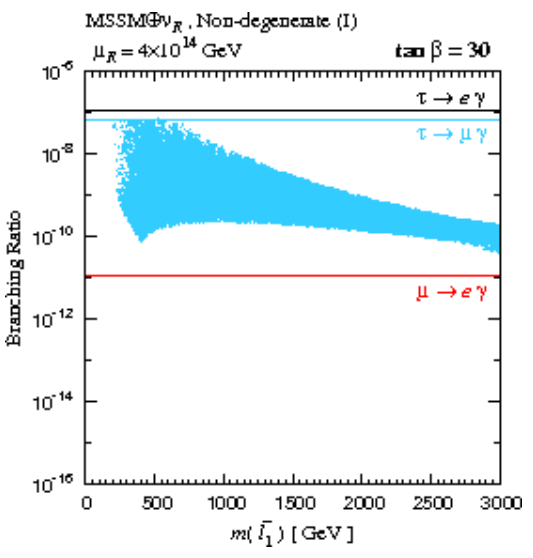

(b)

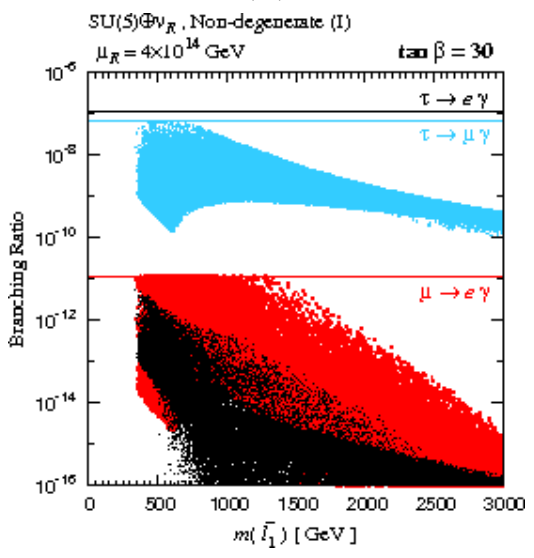

(e)

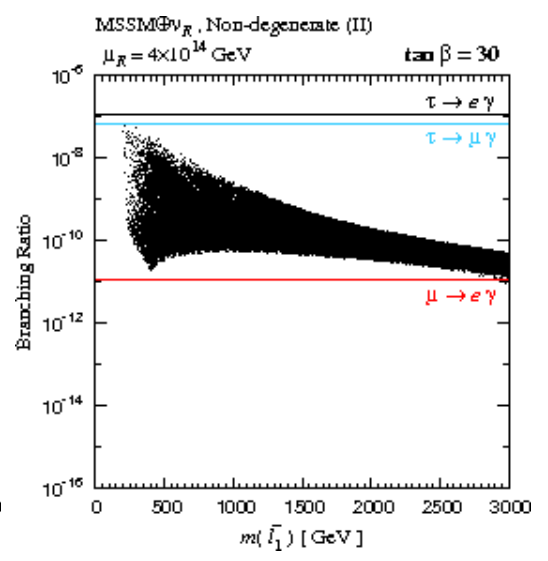

(c)

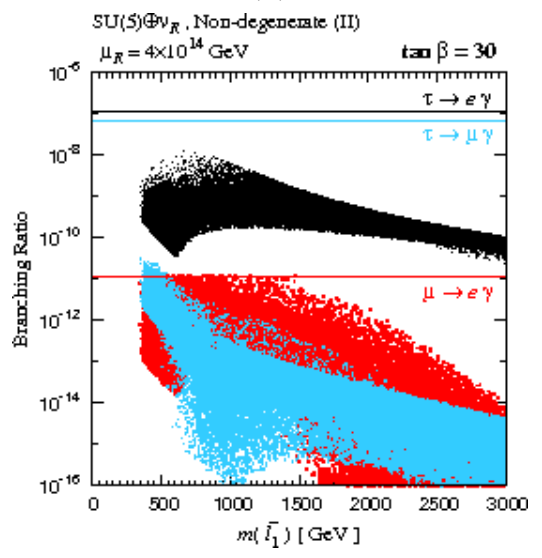

(f)

FIG. 3: (Color online) Branching ratios of lepton flavor violation processes $\mu \rightarrow e \gamma$ (grey/red), $\tau \rightarrow \mu \gamma$ (light-grey/light-blue), and $\tau \rightarrow e \gamma$ (black) as functions of the lightest charged slepton mass $m\left(\tilde{l}_{1}\right)$ for (a)-(c) MSSM with right-handed neutrinos and (d)-(f) SU(5) SUSY GUT with right-handed neutrinos. Horizontal lines denote experimental upper limits. In the plot (b), $\mu \rightarrow e \gamma$ and $\tau \rightarrow e \gamma$ are strongly suppressed. In the plot (c), $\mu \rightarrow e \gamma$ and $\tau \rightarrow \mu \gamma$ are strongly suppressed.

\section{Lepton flavor violating $\mu$ and $\tau$ decays}

There are lepton flavor mixings in the slepton sector of the MSSM with right-handed neutrinos and the SU(5) SUSY GUT with right-handed neutrinos. It comes through the running between the right-handed neutrino mass scale and the cut-off scale where the universal soft breaking mass terms are generated. On the other hand, no such slepton flavor mixings exist in the mSUGRA.

In Fig. 3, the branching ratios of $\mu \rightarrow e \gamma, \tau \rightarrow \mu \gamma$ and $\tau \rightarrow e \gamma$ are displayed as a function 


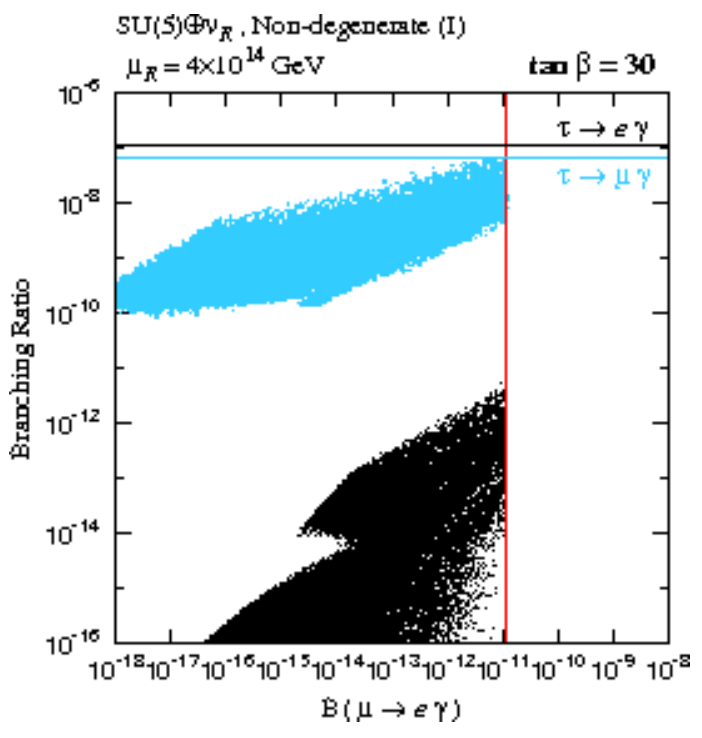

(a)

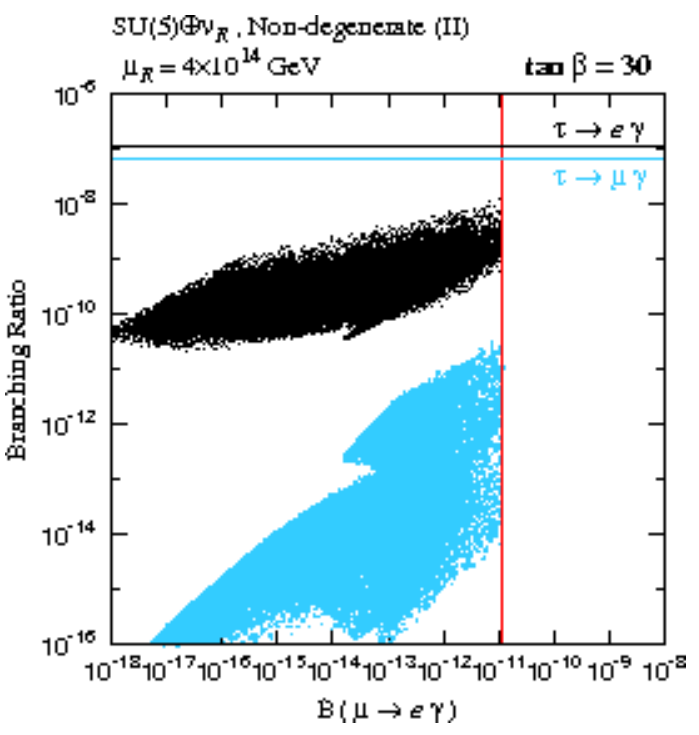

(b)

FIG. 4: (Color online) Correlations between $\mathrm{B}(\tau \rightarrow \mu(e) \gamma)$ and $\mathrm{B}(\mu \rightarrow e \gamma)$. Light-grey (lightblue) and black dots denote $\tau \rightarrow \mu \gamma$ and $\tau \rightarrow e \gamma$, respectively. Experimental upper limits of the branching ratios are shown by horizontal and vertical lines in each plot.

of the lightest charged slepton mass $m\left(\tilde{l}_{1}\right)$ for the MSSM with right-handed neutrinos and the SU(5) SUSY GUT with right-handed neutrinos. For each model, we show the results for three cases of the neutrino Yukawa coupling matrix as explained in Sec. IIA2. The right-handed neutrino mass scale $\mu_{R}$ is taken as $\mu_{R}=4 \times 10^{14} \mathrm{GeV}$, which corresponds to the neutrino Yukawa couplings of $O(1)$. It is known that branching ratios are enhanced by a factor of $\tan ^{2} \beta$ for large values of $\tan \beta$. In the presented plots, we take $\tan \beta=30$ except for the degenerate case in the SU(5) SUSY GUT with right-handed neutrinos, where we show the result for $\tan \beta=10$. When we $\operatorname{take} \tan \beta=30$ for the degenerate case in the SU(5) SUSY GUT with right-handed neutrinos, all the data points in the scanned parameter space are excluded due to the $\mathrm{B}(\mu \rightarrow e \gamma)$ constraint.

We can see that the $\mu \rightarrow e \gamma$ decay rate is enhanced in the degenerate cases. In fact, even for the slepton as heavy as $3 \mathrm{TeV}, \mathrm{B}(\mu \rightarrow e \gamma)$ is close to (or above) the experimental upper limit. After applying the constraint from $\mathrm{B}(\mu \rightarrow e \gamma)$, the branching ratio of $\tau \rightarrow \mu \gamma$ can be $10^{-9}$ at most.

In the non-degenerate $(\mathrm{I})$ case, $\mathrm{B}(\mu \rightarrow e \gamma)$ is suppressed compared to the degenerate cases, so that the constraint is weakened. In particular, there is an approximate electron-number 
conservation in the MSSM with right-handed neutrinos with the Yukawa coupling matrix of the structure Eq. (13), which leads to the suppression of both $\mu \rightarrow e \gamma$ and $\tau \rightarrow e \gamma$. The branching ratio of $\tau \rightarrow \mu \gamma$ can be as large as the current experimental upper limit. In the SU(5) SUSY GUT with right-handed neutrinos, the electron-number conservation is broken by GUT interactions. As a result, $\mathrm{B}(\mu \rightarrow e \gamma)$ can be also as large as the current experimental upper limit. In the non-degenerate (II) cases, the role of $e$ and $\mu$ are interchanged due to the Yukawa structure Eq. (14).

Correlations between $\mathrm{B}(\tau \rightarrow \mu(e) \gamma)$ and $\mathrm{B}(\mu \rightarrow e \gamma)$ in the non-degenerate cases of the SU(5) SUSY GUT with right-handed neutrinos are shown in Fig. 4. Since the MEG experiment can measure $\mathrm{B}(\mu \rightarrow e \gamma)$ down to $10^{-13}$ and the Super $B$ factory can measure $\mathrm{B}(\tau \rightarrow \mu \gamma)$ and $\mathrm{B}(\tau \rightarrow e \gamma)$ of $10^{-9}$, it is possible to distinguish the structure of the slepton flavor mixing if the slepton mass is less than $1 \mathrm{TeV}$.

\section{Quark flavor signals}

We show quark flavor signals in the mSUGRA, SU(5) SUSY GUT with right-handed neutrinos and the $\mathrm{U}(2)$ flavor symmetry models. In the MSSM with right-handed neutrinos, there is no new source of the squark flavor mixing other than the CKM matrix. The effect of the neutrino Yukawa couplings appears in the squark sector only through the renormalization of the Higgs fields. Consequently the flavor structure of the squarks is essentially the same as the mSUGRA case. In fact, we have checked that the plots of the quark flavor signals look similar to those in the mSUGRA, except that the allowed SUSY parameter region is largely affected by the constraints from the LFV processes. That is why we do not show the plots in the MSSM with right-handed-neutrinos here. Quark flavor signals in the SU(5)

SUSY GUT with right-handed neutrinos are affected by the existence of the neutrino Yukawa coupling through the GUT running between $\mu_{P}$ and $\mu_{G}$. Large $b-s$ and $b-d$ mixings in the right-handed down-type squark sector are induced by the large $2-3$ and $1-3$ mixing in the neutrino Yukawa coupling in the non-degenerate (I) and (II) cases, respectively. In the degenerate case, the parameter region with sizable squark mixings is excluded due to the strong $\mu \rightarrow e \gamma$ constraint. The $\mathrm{U}(2)$ model has large 2-3 mixings in both right-handed and left-handed squark sector at the cut-off scale.

Here we show our results on the following observables. 


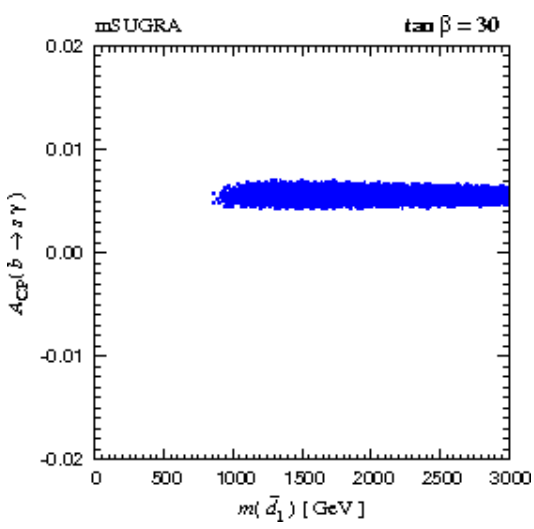

(a)

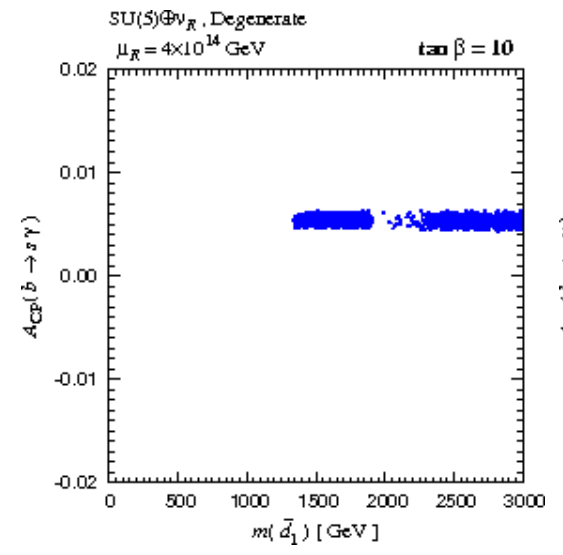

(c)

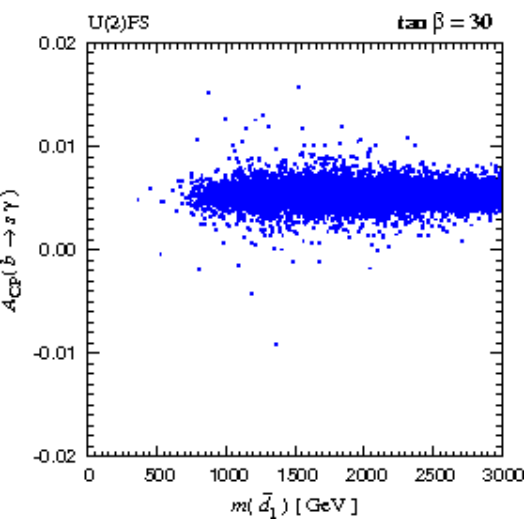

(b)

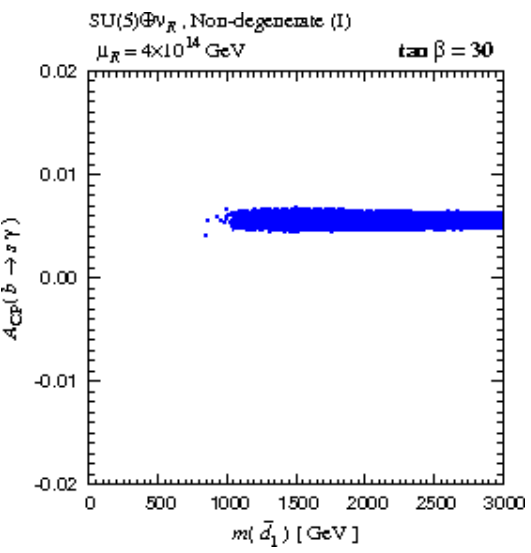

(d)

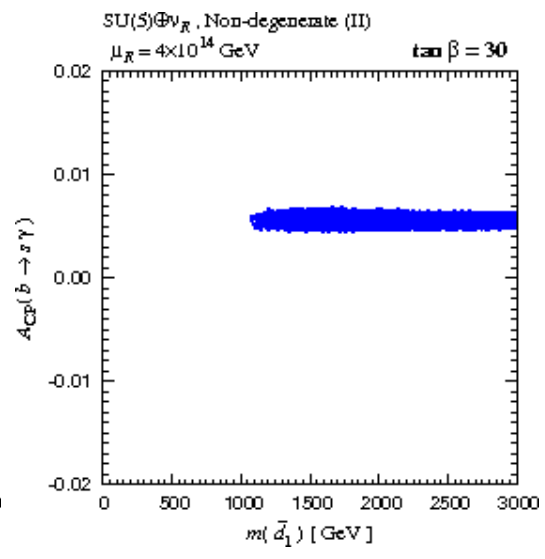

(e)

FIG. 5: The direct CP asymmetry in $b \rightarrow s \gamma$ as functions of the lightest down-type squark mass $m\left(\tilde{d}_{1}\right)$ for (a) mSUGRA, (b) U(2) model and (c)-(e) three cases of the SU(5) SUSY GUT with right-handed neutrinos.

- The direct CP asymmetry in $b \rightarrow s \gamma$ decay (Fig. [5), which is sensitive to the effect of new $\mathrm{CP}$ violating phase in $b \rightarrow s \gamma$ decay amplitude.

- The mixing-induced CP asymmetry in $B_{d} \rightarrow K^{*} \gamma$ (Fig. 6). This asymmetry is enhanced by the $b \rightarrow s \gamma$ decay amplitude with the chirality opposite to the SM one.

- The direct CP asymmetry in $b \rightarrow d \gamma$ decay (Fig. 7), which is sensitive to the effect of new $\mathrm{CP}$ violating phase in $b \rightarrow d \gamma$ decay amplitude.

- The mixing-induced CP asymmetry in $B_{d} \rightarrow \rho \gamma$ (Fig. 8), which is enhanced by the $b \rightarrow d \gamma$ decay amplitude with the chirality opposite to the SM one.

- The mixing-induced CP asymmetry in $B_{d} \rightarrow \phi K_{S}$ decay (Fig. 9). The difference 


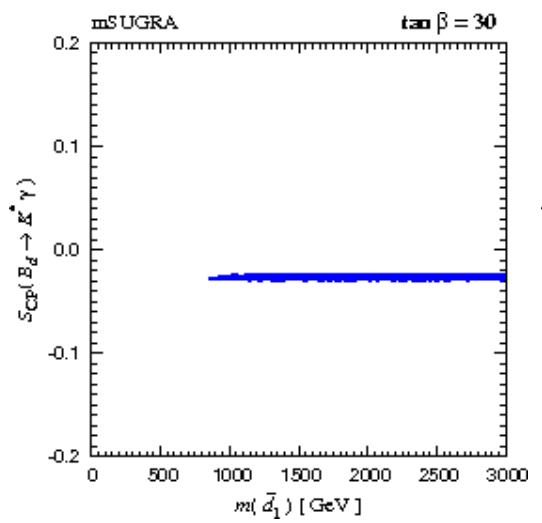

(a)

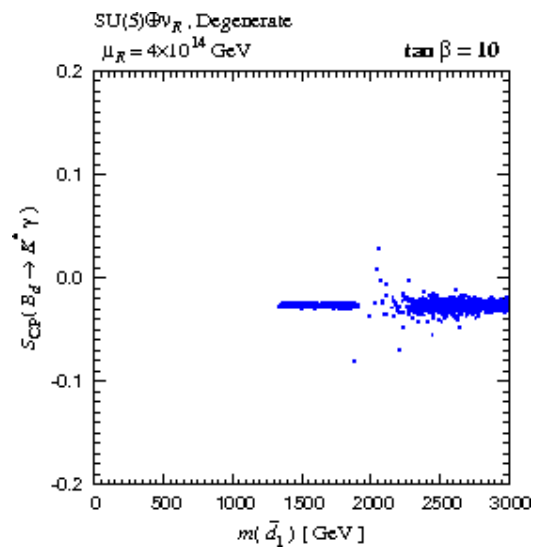

(c)

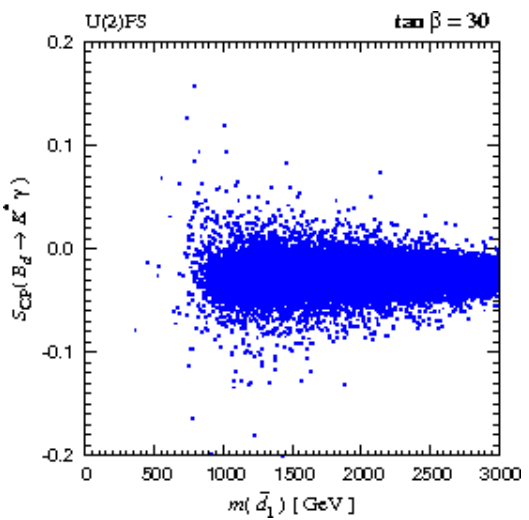

(b)

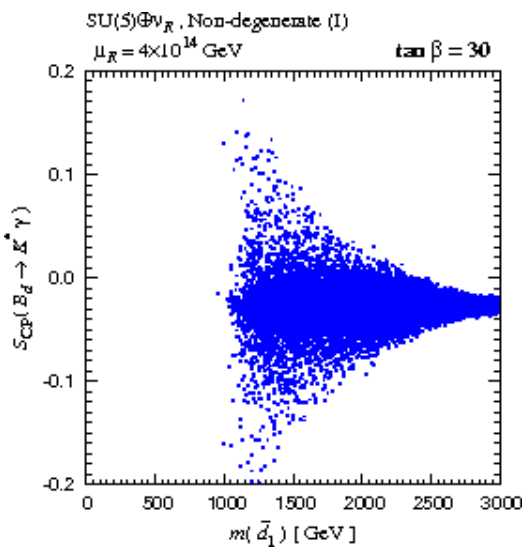

(d)

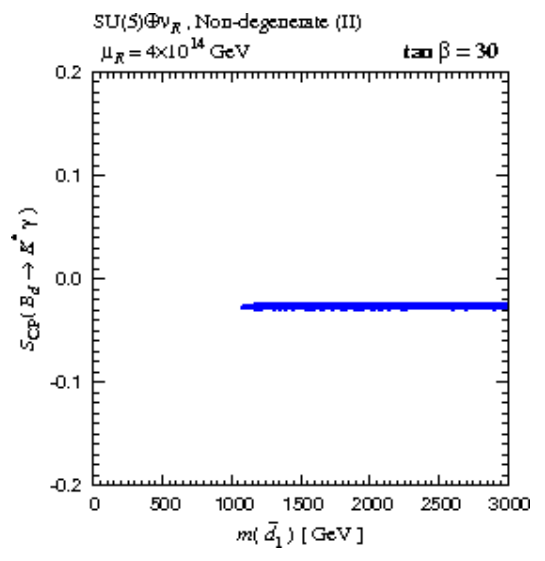

(e)

FIG. 6: The mixing-induced CP asymmetry in $B_{d} \rightarrow K^{*} \gamma$ as functions of $m\left(\tilde{d}_{1}\right)$ for the same parameter sets as those for Fig. [5.

between this quantity and the mixing-induced $\mathrm{CP}$ asymmetry in $B_{d} \rightarrow J / \psi K_{S}$, $\Delta S_{\mathrm{CP}}\left(B_{d} \rightarrow \phi K_{S}\right) \equiv S_{\mathrm{CP}}\left(B_{d} \rightarrow \phi K_{S}\right)-S_{\mathrm{CP}}\left(B_{d} \rightarrow J / \psi K_{S}\right)$, is sensitive to the new $\mathrm{CP}$ violating phase in $b \rightarrow s \bar{s} s$ decay amplitude.

- The mixing-induced CP asymmetry in $B_{s} \rightarrow J / \psi \phi$ decay (Fig. 10), which is affected by the new $\mathrm{CP}$ violating phase in $B_{s}-\bar{B}_{s}$ mixing matrix element.

From Figs. 5, 6, 7, 8, 9] and 10, we can draw the following conclusions. For the mSUGRA case, we do not see significant deviations in any of above observables. In the degenerate case of the SU(5) SUSY GUT with right-handed neutrinos, the parameter region is strongly constrained by the $\mathrm{B}(\mu \rightarrow e \gamma)$ as already discussed. There are some points in which deviations are apparent in $S_{\mathrm{CP}}\left(B_{d} \rightarrow K^{*} \gamma\right), S_{\mathrm{CP}}\left(B_{d} \rightarrow \rho \gamma\right), \Delta S_{\mathrm{CP}}\left(B_{d} \rightarrow \phi K_{S}\right)$ and $S_{\mathrm{CP}}\left(B_{s} \rightarrow J / \psi \phi\right)$. These points could be distinguished by future measurements such as 


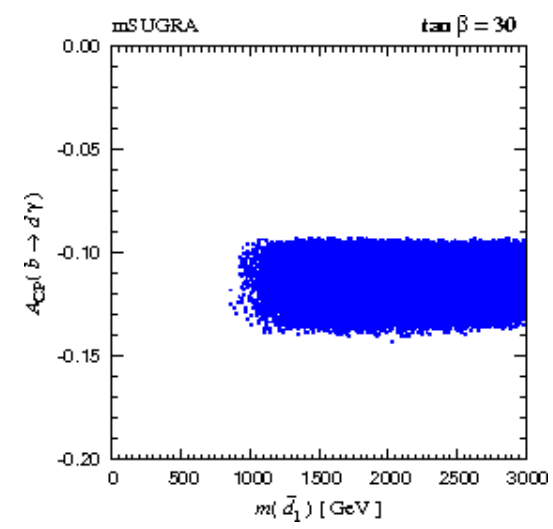

(a)

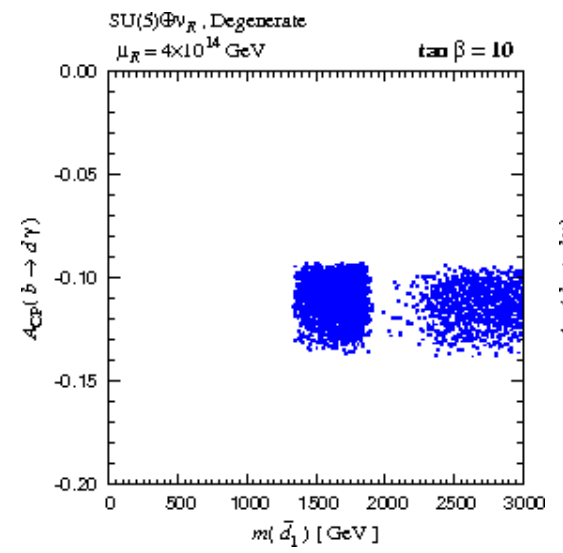

(c)

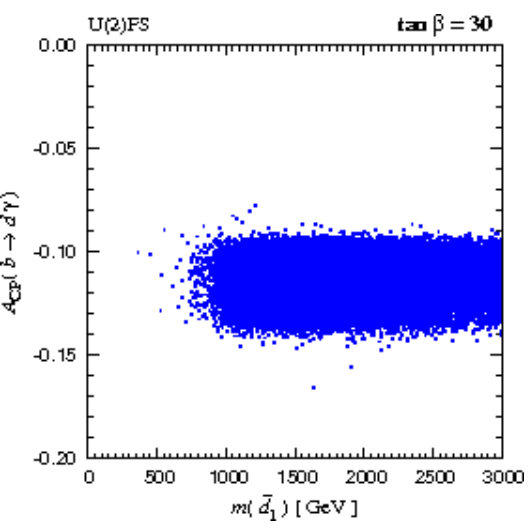

(b)

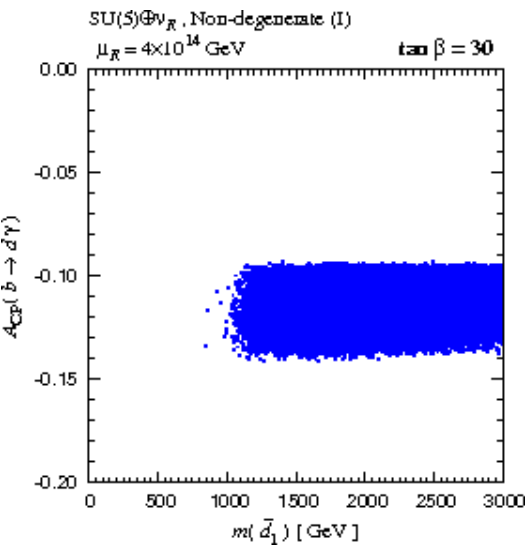

(d)

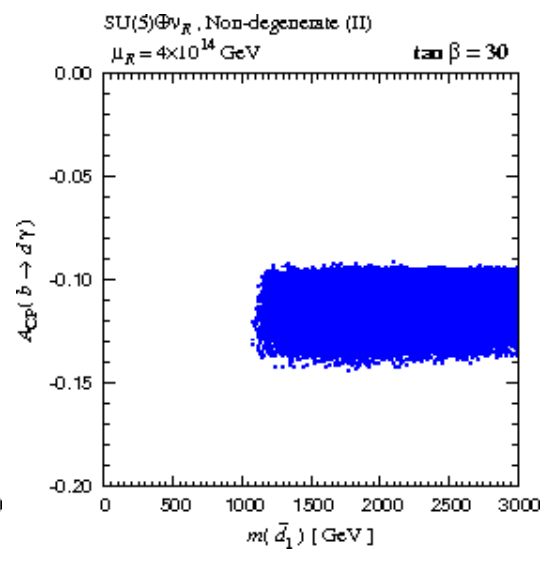

(e)

FIG. 7: The direct CP asymmetry in $b \rightarrow d \gamma$ as functions of $m\left(\tilde{d}_{1}\right)$ for the same parameter sets as those for Fig. 5 .

$\mathrm{LHCb}$, in which the precision in the determination of the phase of $B_{s}-\bar{B}_{s}$ mixing matrix element is expected to be 0.01 radian level [12]. In the non-degenerate (I) case of the SU(5) SUSY GUT with right-handed neutrinos, the SUSY contributions to mixing-induced CP asymmetries in $B_{s} \rightarrow J / \psi \phi, B_{d} \rightarrow K^{*} \gamma$, and $B_{d} \rightarrow \phi K_{S}$ can be significant. On the other hand, in the non-degenerate (II) case of SU(5) SUSY GUT with right-handed neutrinos, there is a significant SUSY contribution to $b \rightarrow d \gamma$ decay amplitude, so that $S_{\mathrm{CP}}\left(B_{d} \rightarrow \rho \gamma\right)$ can be as large as \pm 0.1 . Large SUSY contributions can be found for almost all modes we analyze in the $\mathrm{U}(2)$ model. Only the direct CP asymmetry in $b \rightarrow d \gamma$ does not show any significant deviation from the SM.

The correlation between $\phi_{3}$ and $\Delta m_{B_{s}} / \Delta m_{B_{d}}$ are shown in Fig. 11. $\Delta m_{B_{s}} / \Delta m_{B_{d}}$ is sensitive to the new physics contributions to the $B_{d}-\bar{B}_{d}$ and $B_{s}-\bar{B}_{s}$ mixing matrix 


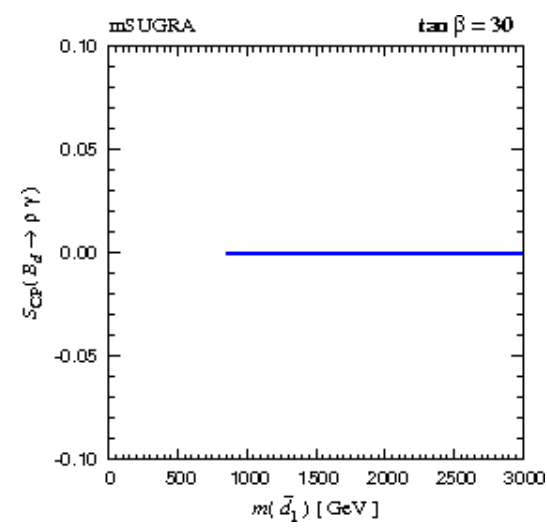

(a)

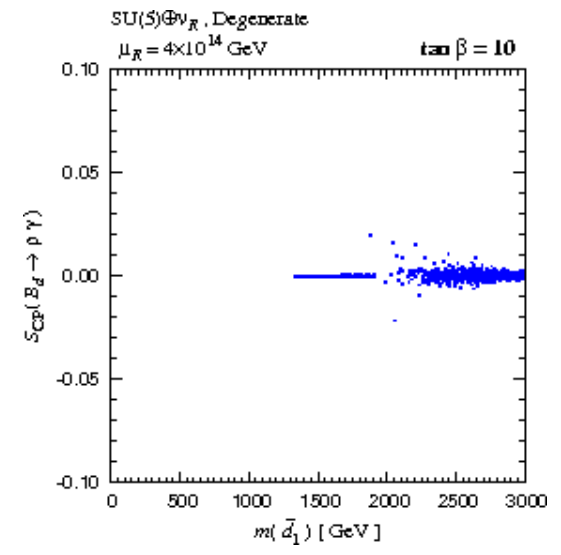

(c)

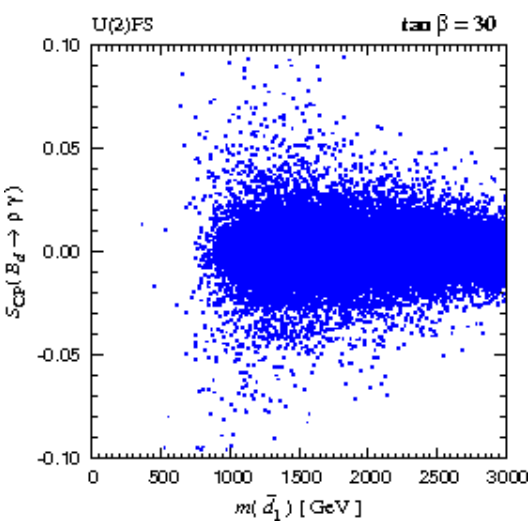

(b)

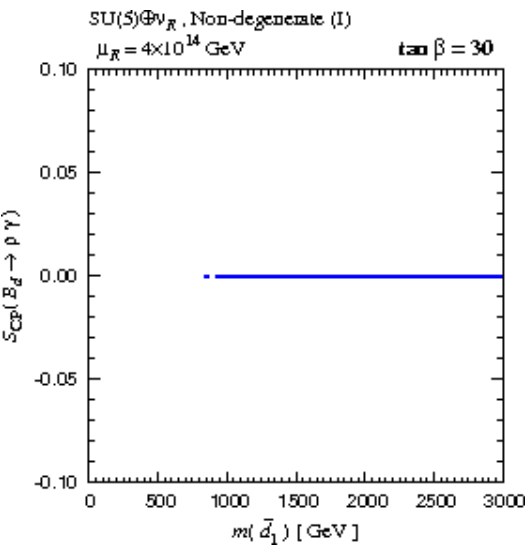

(d)

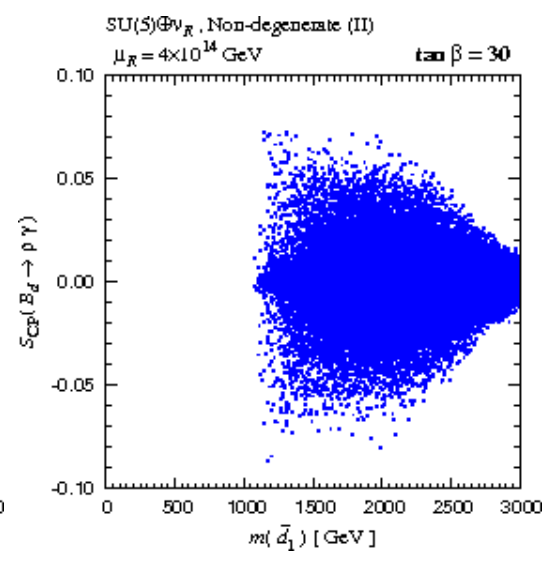

(e)

FIG. 8: The mixing-induced CP asymmetry in $B_{d} \rightarrow \rho \gamma$ as functions of $m\left(\tilde{d}_{1}\right)$ for the same parameter sets as those for Fig. 5 .

elements unless the contributions cancel in the ratio. For the mSUGRA case, the deviation is negligible and the plot in this plane is the same as in the SM. The lower limit of $\phi_{3}$ is determined by the constraint from $\varepsilon_{K}$. In the degenerate case of SU(5) SUSY GUT with right-handed neutrinos, the deviation in the correlation is not so significant. In the nondegenerate cases SU(5) SUSY GUT with right-handed neutrinos and the U(2) model, some deviations appear in the correlation plots. In the non-degenerate (I) case the deviation comes from the SUSY contribution to the $B_{s}-\bar{B}_{s}$ mixing matrix element, while $B_{d}-\bar{B}_{d}$ receive sizable SUSY correction in the non-degenerate (II). In the U(2) model SUSY contributions show up in both matrix elements. In order to identify the deviation in the correlation in future, it is required that the evaluation of $\xi$ parameter by the lattice QCD calculation is significantly improved and that the $\phi_{3}$ is precisely measured from tree-level dominant 


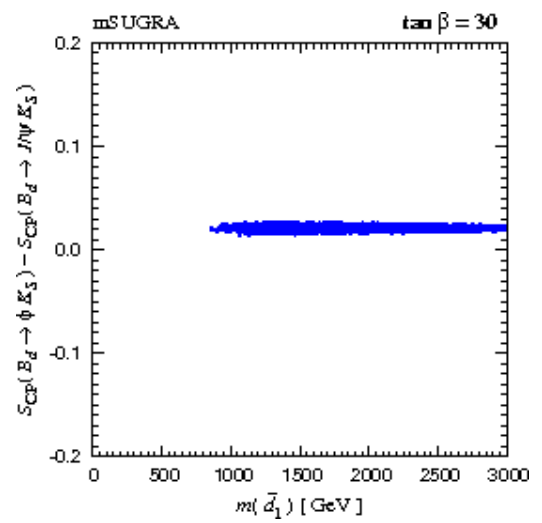

(a)

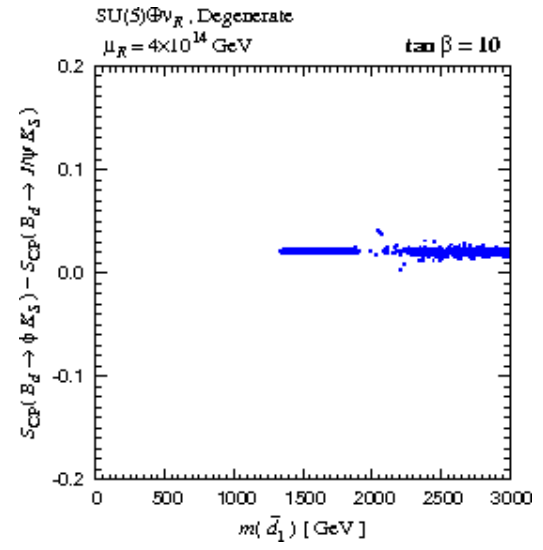

(c)

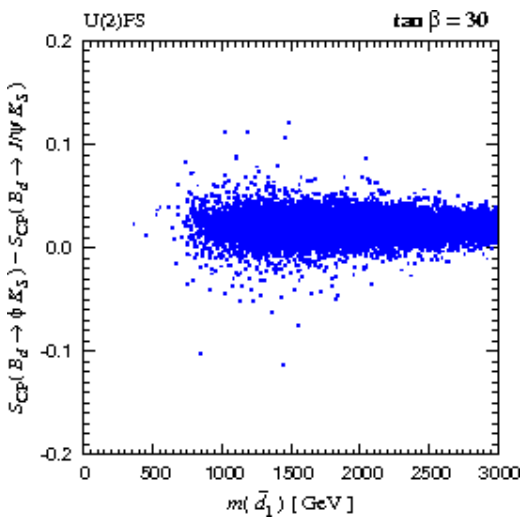

(b)

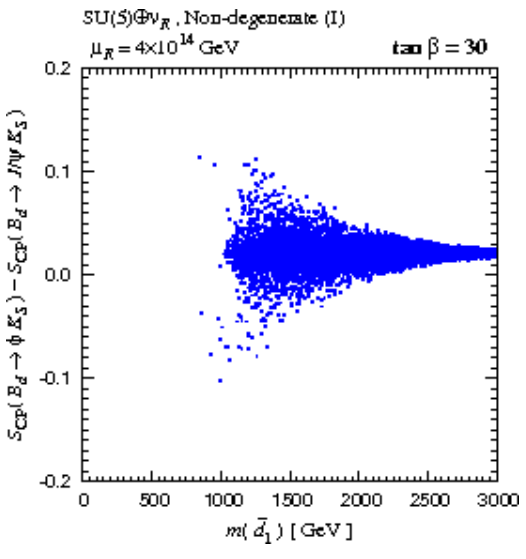

(d)

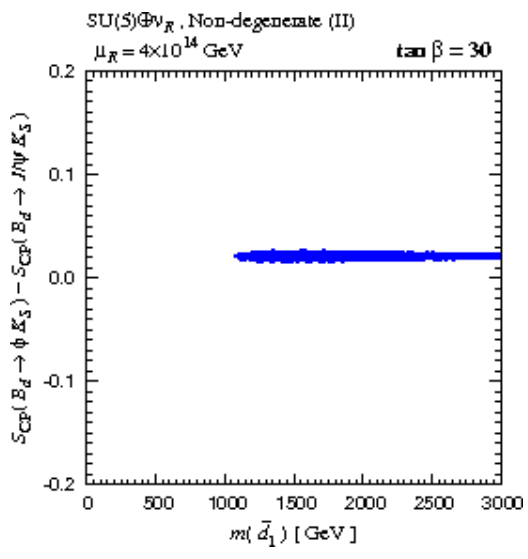

(e)

FIG. 9: The difference between mixing-induced CP asymmetries in the $B_{d} \rightarrow \phi K_{S}$ and $B_{d} \rightarrow$ $J / \psi K_{S}$ modes as functions of $m\left(\tilde{d}_{1}\right)$ for the same parameter sets as those in Fig. 5 .

processes.

We also calculate the branching ratio and the forward-backward asymmetry of $b \rightarrow s l^{+} l^{-}$, which are sensitive to the amplitudes from photon- and $Z$-penguin and box diagrams. In all the cases we consider here, we find the deviations are negligible.

\section{EDM constraints}

We show the EDMs of the neutron, ${ }^{199} \mathrm{Hg}$ and the electron as functions of the lightest down-type squark mass in Fig. 12. Here we use the NDA formula for the neutron EDM. Primary source of these EDMs is the phase of the $A_{0}$, since we fix the phase of the higgsino mass parameter as $\phi_{\mu}=0$ in the present analysis. The EDMs for $\phi_{\mu}=O(1)$ are larger than those for $\phi_{\mu}=0$ by one or two orders of magnitude and easily exceed the experimental upper 


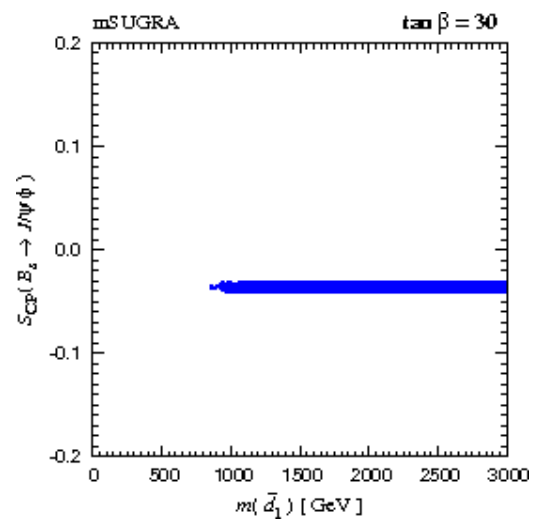

(a)

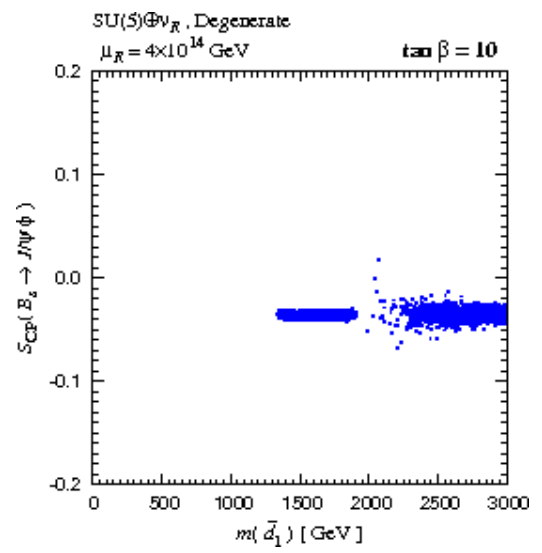

(c)

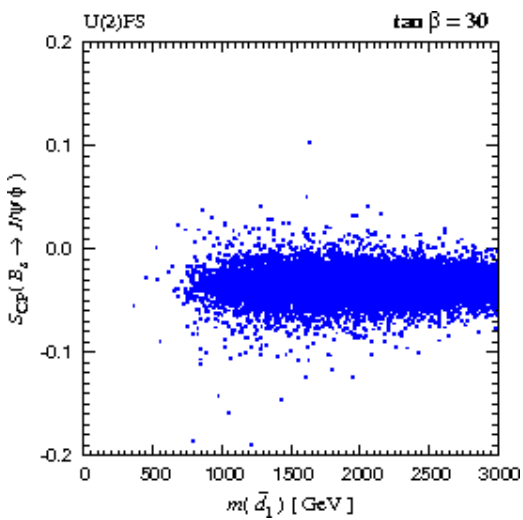

(b)

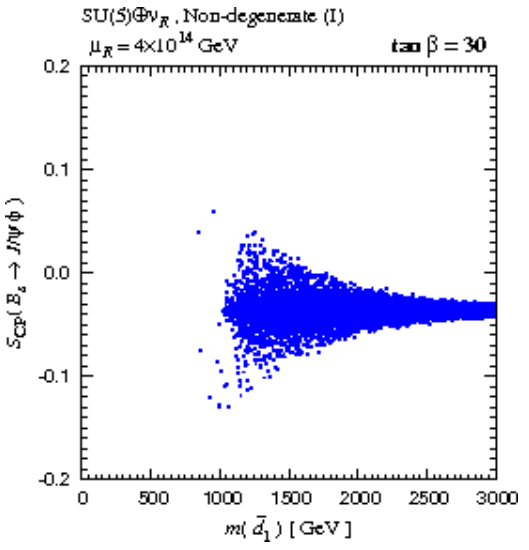

(d)

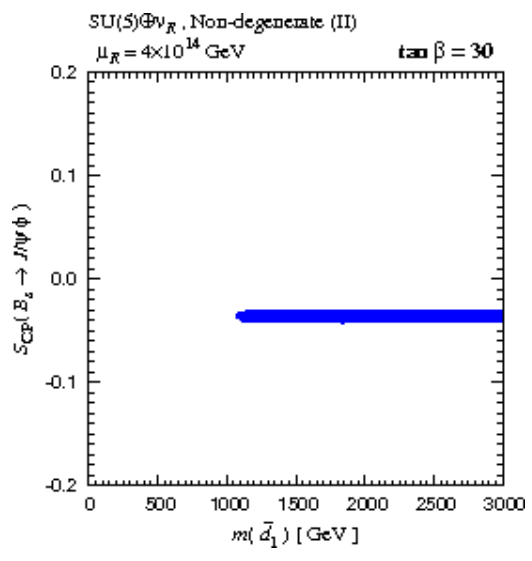

(e)

FIG. 10: Predicted value of the mixing-induced CP asymmetry in $B_{s} \rightarrow J / \psi \phi$ as a function of $m\left(\tilde{d}_{1}\right)$ for the same parameter sets as those for Fig. 5 .

limits in large portions of the parameter space. In the present case, we can see that the upper limit of the electron EDM mainly constrain the parameter space, while the constraints from other two EDMs are slightly weaker.

Let us discuss how the possible quark and lepton flavor signals change if we use the formula for the neutron EDM based on the chiral perturbation theory, as mentioned in Sec. III A 4. The main difference between the NDA and ChPT formulae is the treatment of the strange quark (chromo-)EDM. In ChPT the contribution from the strange quark is taken into account, while it is simply neglected in NDA. In the non-degenerate (I) of the SU(5) SUSY GUT with right-handed neutrinos and the U(2) cases, the $2-3$ generation mixings and $\mathrm{CP}$ violating phases exist in both left- and right-handed down-type squark mass matrices, which enhance the chromo-EDM of the strange quark. Therefore, the SUSY contributions to the $\mathrm{CP}$ asymmetries in $b \rightarrow s$ decays correlate to the chromo-EDM of the 


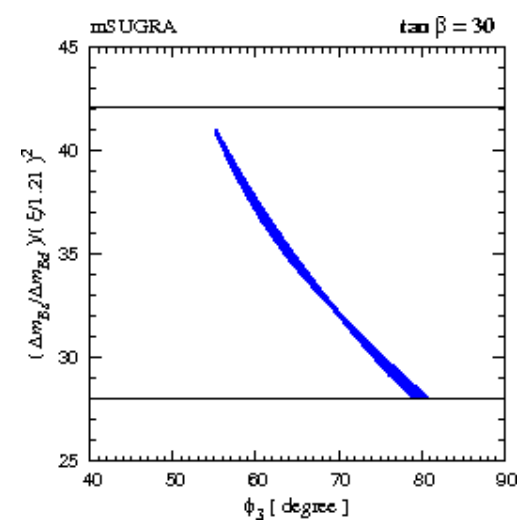

(a)

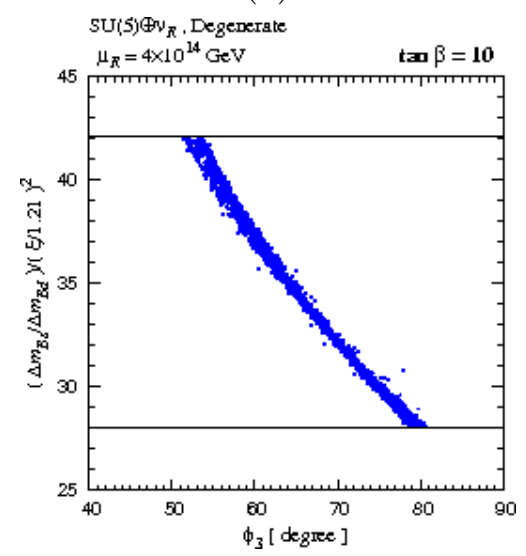

(c)

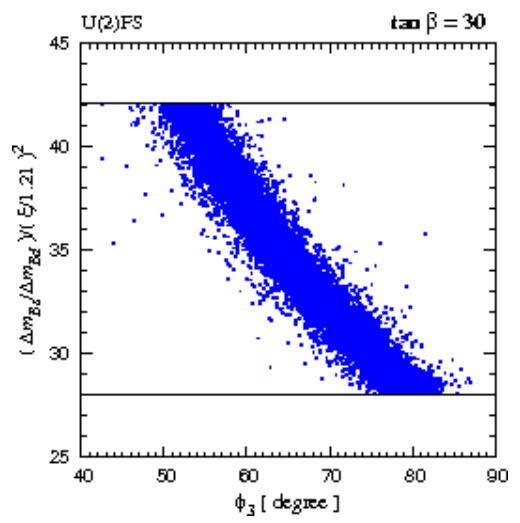

(b)

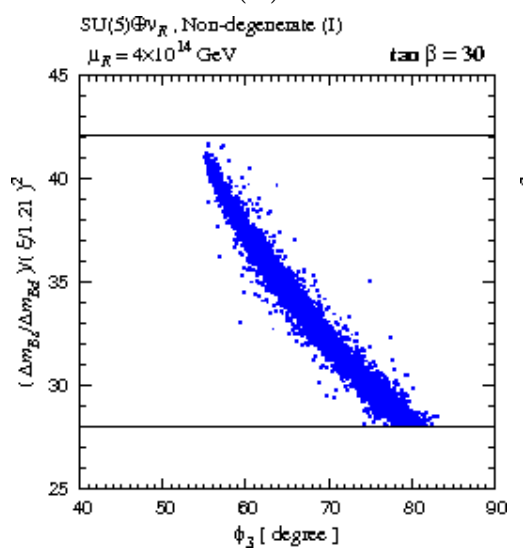

(d)

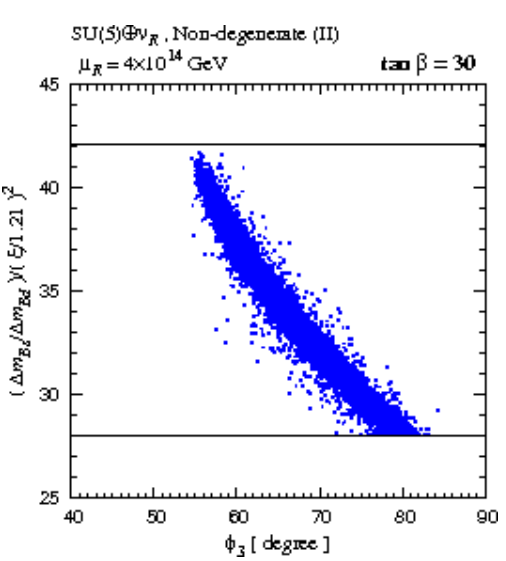

(e)

FIG. 11: Correlation between $\phi_{3}$ and $\Delta m_{B_{s}} / \Delta m_{B_{d}}$ for the same parameter sets as those in Fig. 5 .

strange quark.

We show the plots of the neutron EDM calculated by the ChPT formula [33] in Fig. 13. It can be seen that the ChPT formula leads to typically $1-2$ orders of magnitude larger value of the neutron EDM than the NDA formula does, in the $m\left(\tilde{d}_{1}\right) \sim 1 \mathrm{TeV}$ region. Therefore, a larger portion of the parameter space with new CP violating phases is excluded if we adopt the ChPT formula for the evaluation of the neutron EDM, and possible deviations in the $\mathrm{CP}$ violation observables are also affected.

In Fig. 14, we show the correlations between the neutron EDM calculated by the ChPT formula and the CP asymmetries $S_{\mathrm{CP}}\left(B_{d} \rightarrow K^{*} \gamma\right), \Delta S_{\mathrm{CP}}\left(B_{d} \rightarrow \phi K_{S}\right)$ and $S_{\mathrm{CP}}\left(B_{s} \rightarrow J / \psi \phi\right)$. The correlations between the neutron EDM and the LFV decay branching ratios are also shown. We show the correlation plots for the $\mathrm{U}(2)$ model in Fig. 15, In particular for the non-degenerate (I) of the SU(5) SUSY GUT with right-handed neutrinos, the parameter region with large deviations of $\Delta S_{\mathrm{CP}}\left(B_{d} \rightarrow \phi K_{S}\right)$ and $S_{\mathrm{CP}}\left(B_{s} \rightarrow J / \psi \phi\right)$ is excluded if we 


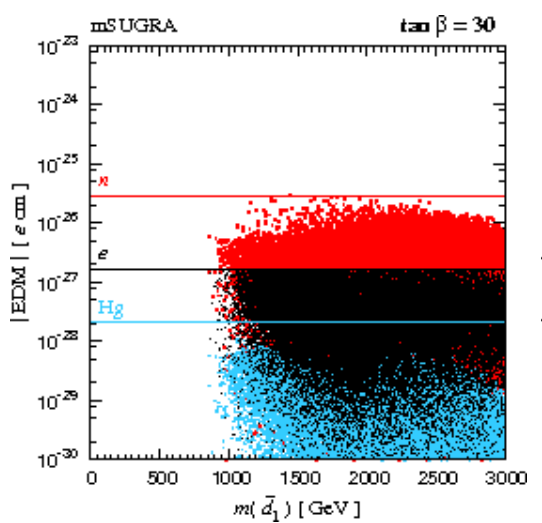

(a)

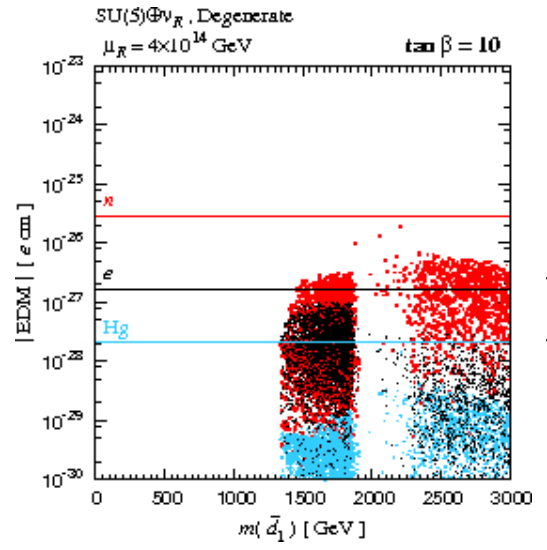

(c)

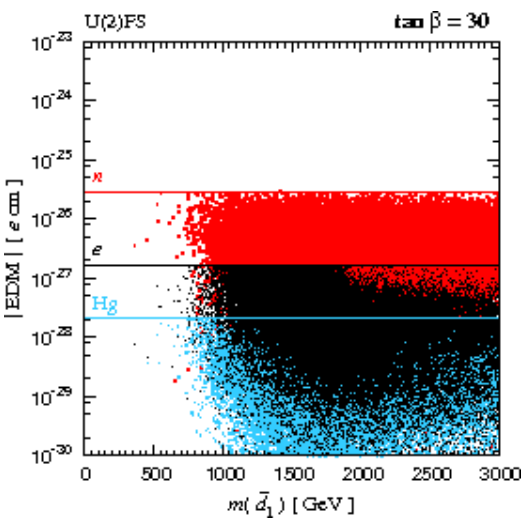

(b)

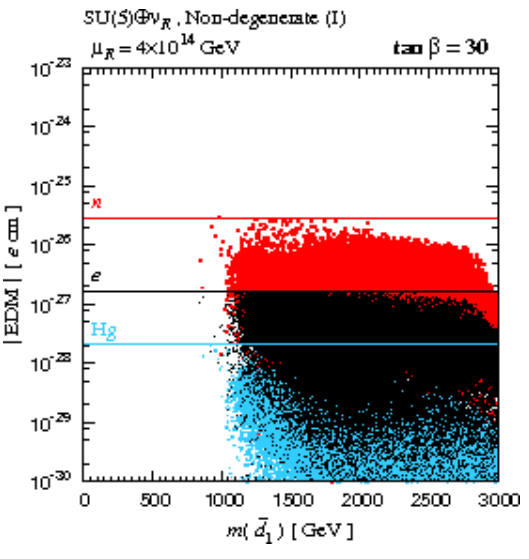

(d)

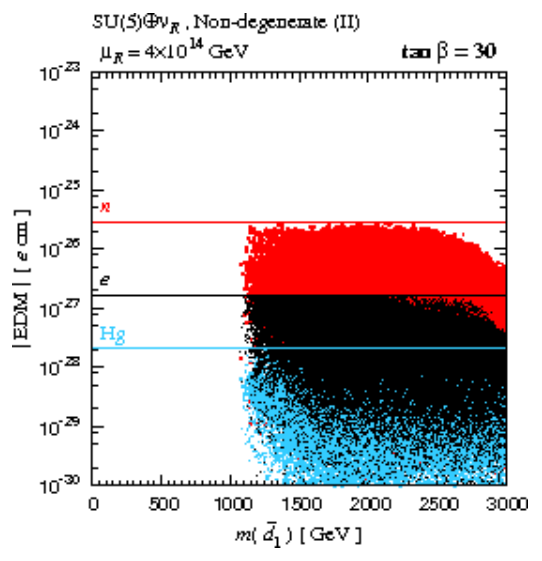

(e)

FIG. 12: (Color online) Electric dipole moments of the neutron (grey/red), ${ }^{199} \mathrm{Hg}$ (light-grey/lightblue) and the electron (black) as functions of $m\left(\tilde{d}_{1}\right)$ for the same parameter sets as those in Fig. 5. Horizontal lines show the experimental upper limits. The neutron EDM is calculated by the NDA formula.

adopt the ChPT formula for the evaluation of the neutron EDM. On the other hand, large deviations remain for other cases.

\section{Summary of results and experimental prospects}

There are good experimental prospects for future improvements in the observables considered above. From recent study of Super $B$ Factories [65], the precision of determination for $50-75 \mathrm{ab}^{-1}$ is $0.02-0.03$ for $S_{\mathrm{CP}}\left(B_{d} \rightarrow K^{*} \gamma\right), 0.08-0.12$ for $S_{\mathrm{CP}}\left(B_{d} \rightarrow \rho \gamma\right)$ and 0.02-0.03 for $S_{\mathrm{CP}}\left(B_{d} \rightarrow \phi K_{S}\right)$ for mixing-induced CP asymmetries. For the direct CP asymmetries of the radiative $B$ decays, the expected sensitivity reach 0.004 for $A_{\mathrm{CP}}(b \rightarrow s \gamma)$ and 0.01 for 


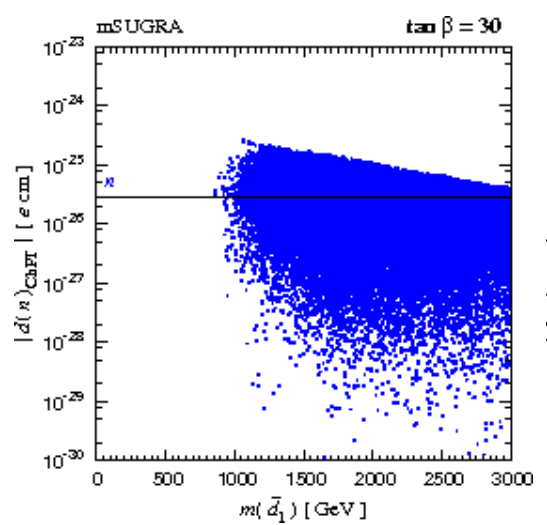

(a)

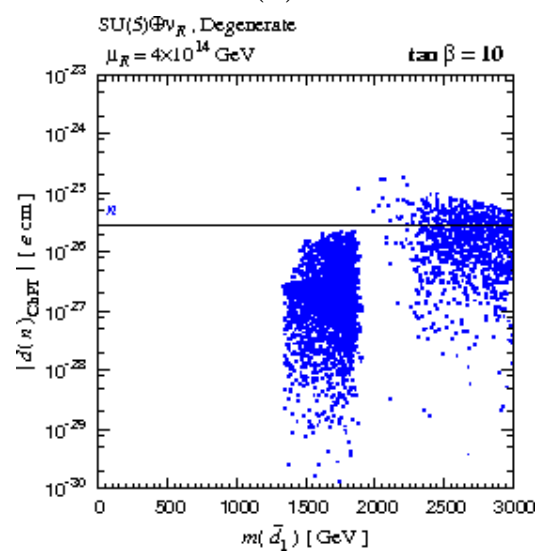

(c)

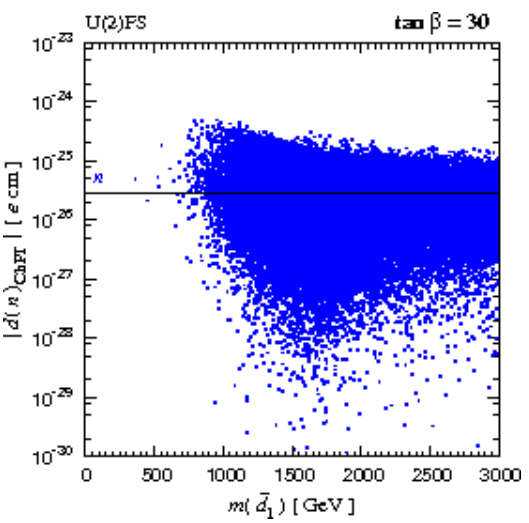

(b)

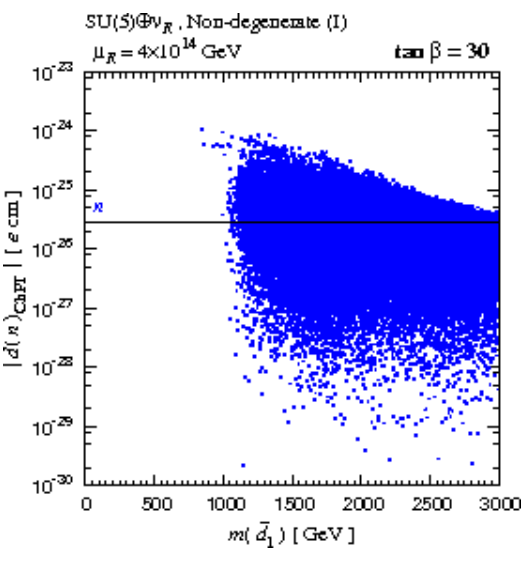

(d)

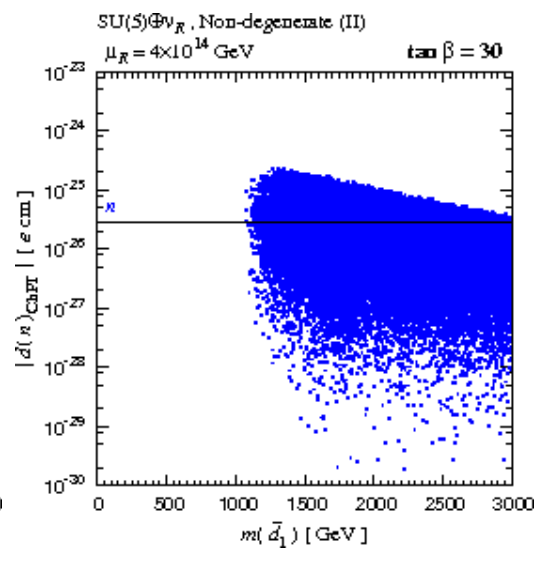

(e)

FIG. 13: The neutron EDM calculated by the ChPT formula for the same parameter sets as those in Fig. 12 ,

$A_{\mathrm{CP}}(b \rightarrow(s+d) \gamma)$. The CP asymmetry of $B_{s} \rightarrow J / \psi \phi$ mode is determined up to 0.01 from $\mathrm{LHCb}$ with $10 \mathrm{fb}^{-1}[12]$. The precision of the $\phi_{3}$ determination is expected at $2.4^{\circ}$ for $\mathrm{LHCb}$ at $10 \mathrm{fb}^{-1}$ [12], and further improvement is expected at Super $B$ Factory. In order to extract new physics effect from the correlation between $\Delta m_{B_{s}} / \Delta m_{B_{d}}$ and $\phi_{3}$ we need to improve the determination of $\xi$ factor up to a percent level. The $\mu \rightarrow e \gamma$ branching ratio will be searched for at the level of $10^{-13}$ level at the MEG experiment. Current upper bounds of $\mathrm{B}(\tau \rightarrow \mu \gamma)$ and $\mathrm{B}(\tau \rightarrow e \gamma)$ are $6.8 \times 10^{-8}$ and $1.1 \times 10^{-7}$, respectively, at the $B$ factory experiments, and future improvement by $1-2$ orders of magnitude is expected at Super $B$ factory.

Comparing with these prospects, we can determine the significance of the deviations observed in Figs. 311, Our results of lepton and quark flavor signals are summarized in Table IV. We list various quark flavor signals in $b-s$ and $b-d$ transition for the mSUGRA, 


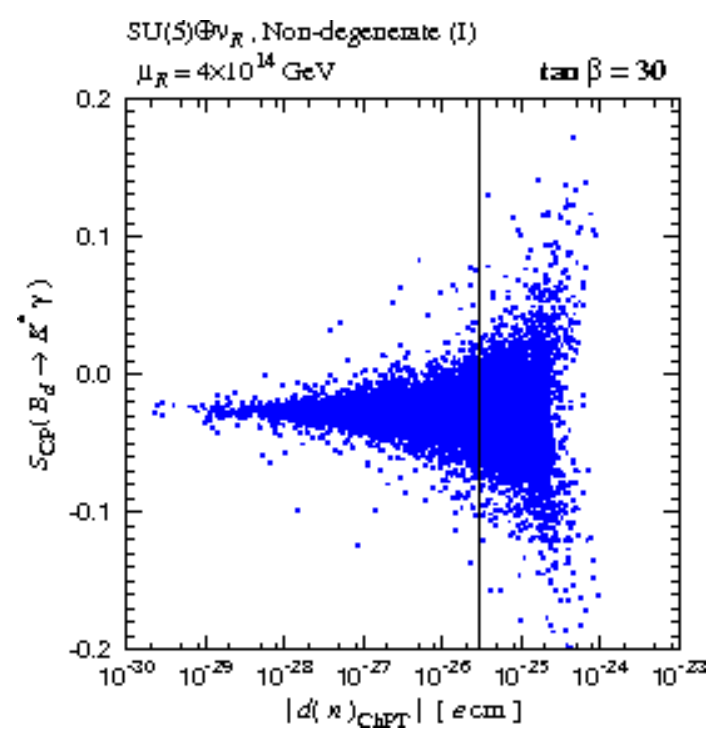

(a)

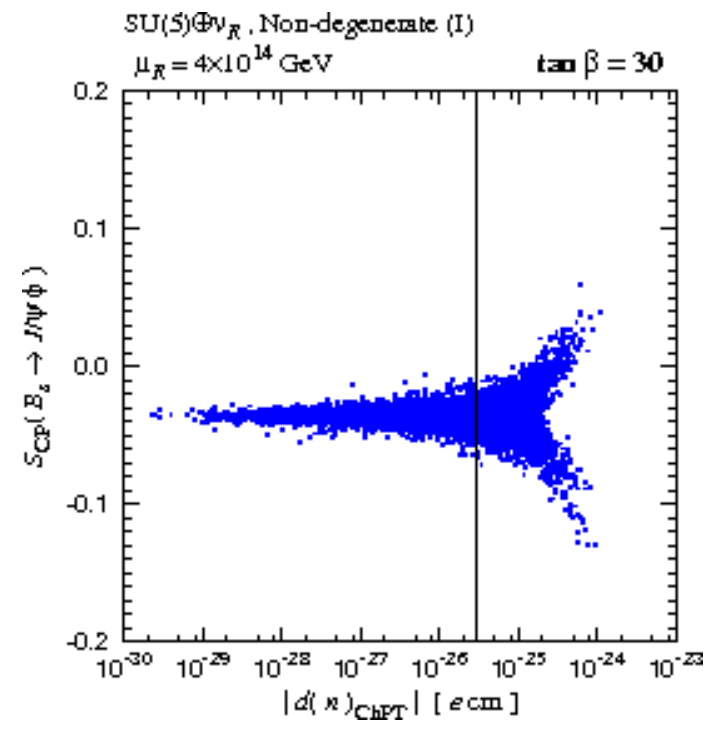

(c)

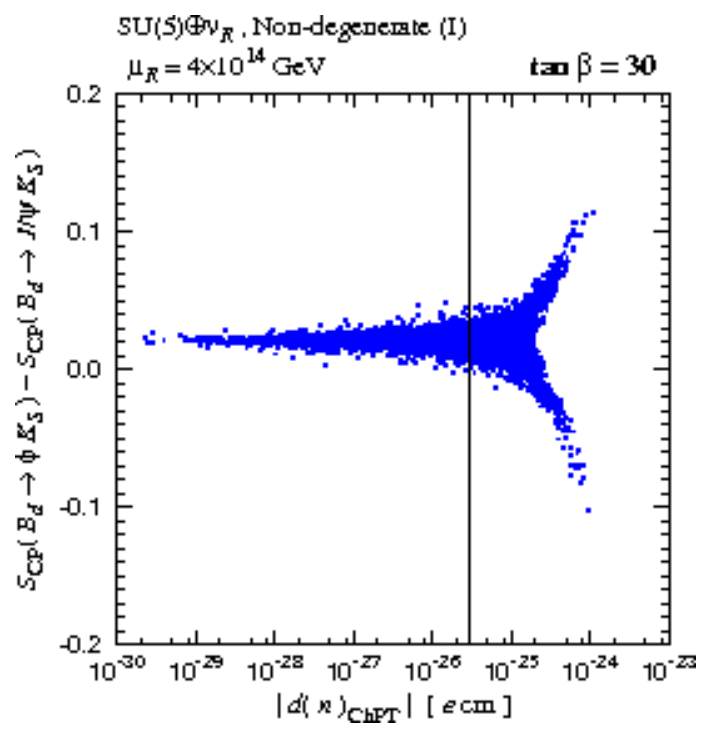

(b)

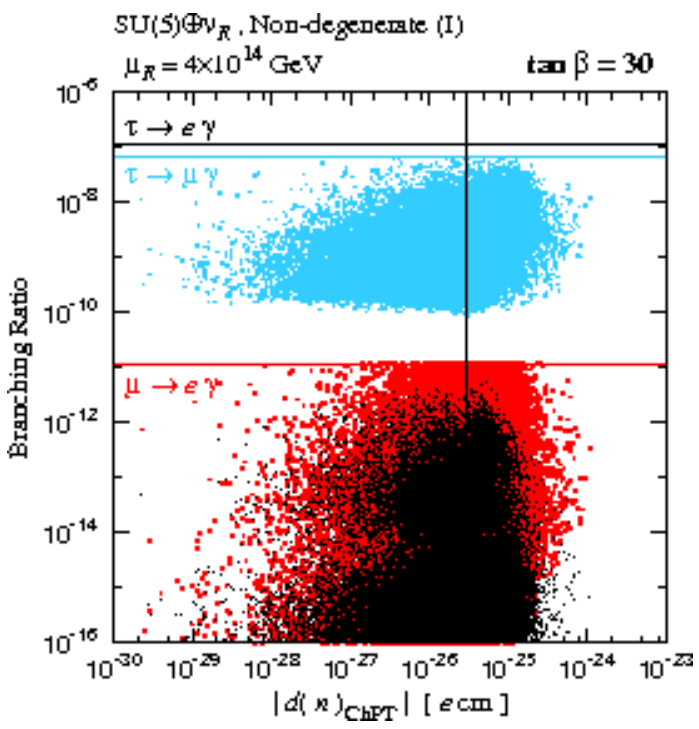

(d)

FIG. 14: (Color online) The CP asymmetries in $b \rightarrow s$ decays and the LFV branching ratios as functions of the neutron EDM given by the chiral perturbation formula in the non-degenerate (I) case of SU(5) SUSY GUT with right-handed neutrinos.

three cases of MSSM with right-handed neutrinos, and SU(5) SUSY GUT with right-handed neutrinos, and the $\mathrm{U}(2)$ flavor symmetry model. The $\mu$ and $\tau$ LFV processes are also included for the cases except for the $\mathrm{U}(2)$ model. The observable with a mark $\sqrt{ }$ indicates that a large deviation is expected. The mark $\bullet$ means that there are some points that the deviation could be identified with future improvements of experimental measurements 


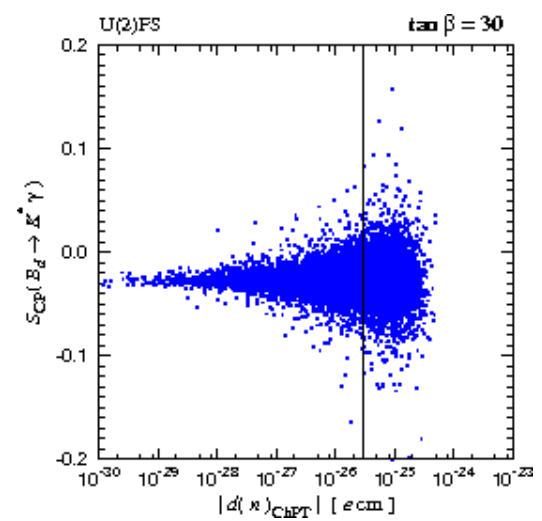

(a)

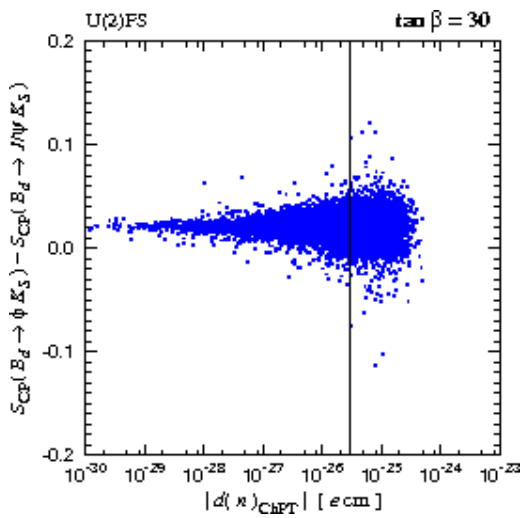

(b)

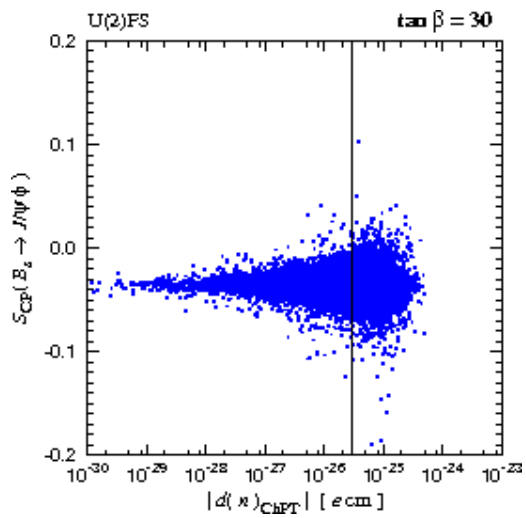

(c)

FIG. 15: The CP asymmetries in $b \rightarrow s$ decays as functions of the neutron EDM given by the chiral perturbation formula in the $\mathrm{U}(2)$ flavor symmetry model.

and/or theoretical understanding of uncertainty. From the table, we can see that significant flavor signals are expected in the lepton sector for the MSSM with right-handed neutrinos and the SU(5) SUSY GUT with right-handed neutrinos. These lepton flavour violation signals depend on the texture of the neutrino Yukawa coupling matrix, i.e. $\tau \rightarrow \mu \gamma$ can be large in the non-degenerate (I) cases and $\tau \rightarrow e \gamma$ can be large in the non-degenerate (II) cases while satisfying the present experimental bound on $\mu \rightarrow e \gamma$. In the degenerate cases, $\mu \rightarrow e \gamma$ is the most promising mode among these three lepton flavour violation processes. In the SU(5) SUSY GUT with right-handed neutrinos, in addition to the above texture dependent signals, $\mu \rightarrow e \gamma$ can be enhanced as large as the present experimental bound due to GUT interactions even in the non-degenerate (I) and (II) cases. As for the quark flavor signals, we can expect that significant $\mathrm{CP}$ violating asymmetries in $b \rightarrow s$ and $b \rightarrow d$ transitions in the SU(5) SUSY GUT with right-handed neutrinos and in the U(2) model. The pattern of the deviations from the SM predictions also depends on the texture of the neutrino Yukawa coupling matrix in the SU(5) SUSY GUT with right-handed neutrinos. Examining the pattern of deviations from the SM in the quark and lepton flavor signals, we can gain insights on the flavor structure in the SUSY models.

In addition to experimental progress, it is important to reduce theoretical uncertainties to identify the deviations. In particular, theoretical issue to predict mixing-induced CP asymmetries in $B_{d} \rightarrow K^{*} \gamma$ [66] and $B_{d} \rightarrow \phi K_{S}$ [67] modes within the SM need to be clarified because the deviation we expected is up to $10 \%$ level. 


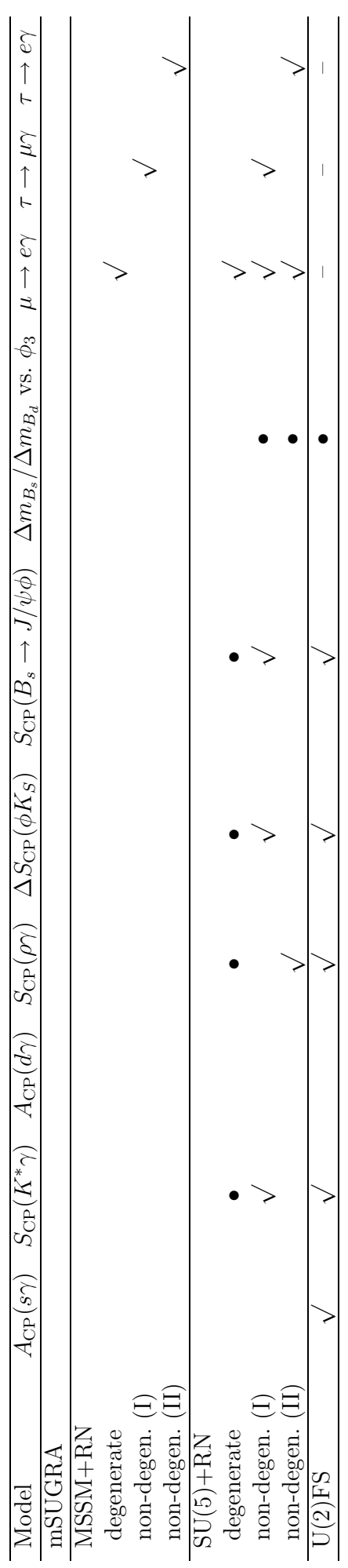

TABLE IV: Summary of expected flavor signals for each model. The observable with a mark $\sqrt{ }$ indicates that a large deviation is expected. The mark $\bullet$ means that there are some points that the deviation could be identified with future improvements of experimental measurements and/or theoretical understanding of uncertainty. We do not consider LFV processes for the U(2)FS model $(-)$. 
In this paper we do not include the heavy Higgs exchange contributions to various FCNC and LFV processes. These contributions are known to play an important role for particular cases of SUSY parameter sets due to large corrections to Yukawa coupling constants through SUSY loop diagrams [68]. The relevant parameter set corresponds to large values of $\tan \beta$ and relatively small values of heavy Higgs boson masses with large values of $\mu$. The Higgs exchange contribution induces drastic effects in processes like $B_{s} \rightarrow \mu^{+} \mu^{-}$and $b \rightarrow s l^{+} l^{-}$ 69] especially for a large value of $\tan \beta(=50-60)$ because of a high power dependence of $\tan \beta$. In some restricted parts of our analysis, we may have additional flavor signals due to the Higgs exchange effects.

\section{CONCLUSIONS}

We have analyzed quark flavor signals associated with $b \rightarrow s$ and $b \rightarrow d$ transitions and lepton flavor violations in various cases of supersymmetric models. Extensive study is carried out in terms of observables for representative SUSY models. Our result is summarized in Table IV. We have improved computational methods and updated phenomenological constraints from our works in previous publications. The most important effect is the inclusion of the constraint from the $B_{s}-\bar{B}_{s}$ mixing from recent Tevatron experiments. The maximum deviation for various $b \rightarrow s$ transition processes turn out to be $10 \%$ level, compared to the previous results where the deviation at the level of $50 \%$ was possible. In this work, we also present predictions of tau lepton flavor processes. Under the constraint of $\mu \rightarrow e \gamma$, the tau LFV processes are promising to look for new physics effects, which are also related to $b \rightarrow s$ and $b \rightarrow d$ transition processes in SUSY GUT models. The pattern of deviation from the SM prediction provides us with an important clue on physics determining the structure of the SUSY breaking sector, and a future $B$ factory plays a central role in such investigation along with on-going flavor experiments such as MEG and LHCb.

\section{Acknowledgments}

The work of T. G. and Y. O. is supported in part by the Grant-in-Aid for Science Research, Ministry of Education, Culture, Sports, Science and Technology, Japan, No. 16081211. The work of Y. O. is supported in part by the Grant-in-Aid for Science Research, Ministry of 
Education, Culture, Sports, Science and Technology, No. 17540286. The work of T. S. is supported in part by the INFN under the program "Fisica Astroparticellare", and by the Italian MIUR (Internazionalizzazione Program). The numerical calculations were carried out in part on Altix3700 BX2 at YITP in Kyoto University.

[1] N. Cabibbo, Phys. Rev. Lett. 10, 531 (1963); M. Kobayashi and T. Maskawa, Prog. Theor. Phys. 49, 652 (1973).

[2] Y. Ashie et al. [Super-Kamiokande Collaboration], Phys. Rev. D 71, 112005 (2005) arXiv:hep-ex/0501064; J. Hosaka et al. [Super-Kamkiokande Collaboration], Phys. Rev. D 73, 112001 (2006) arXiv:hep-ex/0508053; B. Aharmim et al. [SNO Collaboration], Phys. Rev. C 75, 045502 (2007) arXiv:nucl-ex/0610020]; M. Altmann et al. [GNO Collaboration], Phys. Lett. B 616, 174 (2005) arXiv:hep-ex/0504037; W. Hampel et al. [GALLEX Collaboration], Phys. Lett. B 447, 127 (1999); J. N. Abdurashitov et al. [SAGE Collaboration], J. Exp. Theor. Phys. 95, 181 (2002) [Zh. Eksp. Teor. Fiz. 122, 211 (2002)] arXiv:astro-ph/0204245.

[3] S. Yamamoto et al. [K2K Collaboration], Phys. Rev. Lett. 96, 181801 (2006) arXiv:hep-ex/0603004; M. H. Ahn et al. [K2K Collaboration], Phys. Rev. D 74, 072003 (2006) arXiv:hep-ex/0606032.

[4] T. Araki et al. [KamLAND Collaboration], Phys. Rev. Lett. 94, 081801 (2005) arXiv:hep-ex/0406035.

[5] M. Apollonio et al. [CHOOZ Collaboration], Eur. Phys. J. C 27, 331 (2003) arXiv:hep-ex/0301017]; A. Piepke [Palo Verde Collaboration], Prog. Part. Nucl. Phys. 48, 113 (2002).

[6] ATLAS Collaboration, Technical Design Report, CERN/LHCC/99-15 (1999); A. Ball, M. Della Negra, A. Petrilli and L. Foa [CMS Collaboration], J. Phys. G 34, 995 (2007).

[7] For reviews, see H. P. Nilles, Phys. Rept. 110, 1 (1984); H. E. Haber and G. L. Kane, Phys. Rept. 117, 75 (1985); S. P. Martin, arXiv:hep-ph/9709356. D. J. H. Chung, L. L. Everett, G. L. Kane, S. F. King, J. D. Lykken and L. T. Wang, Phys. Rept. 407, 1 (2005) arXiv:hep-ph/0312378.

[8] J. R. Ellis and D. V. Nanopoulos, Phys. Lett. B 110, 44 (1982).

[9] J. R. Ellis, S. Ferrara and D. V. Nanopoulos, Phys. Lett. B 114, 231 (1982). 
[10] L. M. Barkov et al., PSI Proposal R-99-05 (1999); S. Ritt [MEG Collaboration], Nucl. Phys. Proc. Suppl. 162, 279 (2006).

[11] S. Amato et al. [LHCb Collaboration], CERN-LHCC-98-04, CERN-LHCC-P-4 (1998).

[12] T. Nakada, talk given at Conference on Supersymmetry in 2010's, June 20-22, 2007, Hokkaido University, Sapporo, Japan.

[13] A. G. Akeroyd et al. [SuperKEKB Physics Working Group], arXiv:hep-ex/0406071; S. Hashimoto et al., KEK-REPORT-2004-4, Jun 2004.

[14] M. Bona et al., arXiv:0709.0451 [hep-ex].

[15] J. L. Hewett et al., arXiv:hep-ph/0503261.

[16] F. Gabbiani and A. Masiero, Nucl. Phys. B 322, 235 (1989); I. I. Y. Bigi and F. Gabbiani, Nucl. Phys. B 352, 309 (1991). J. S. Hagelin, S. Kelley and T. Tanaka, Nucl. Phys. B 415, $293(1994)$.

[17] F. Gabbiani, E. Gabrielli, A. Masiero and L. Silvestrini, Nucl. Phys. B 477, 321 (1996) arXiv:hep-ph/9604387]; M. Ciuchini et al., JHEP 9810, 008 (1998) arXiv:hep-ph/9808328]; T. Besmer, C. Greub and T. Hurth, Nucl. Phys. B 609, 359 (2001) arXiv:hep-ph/0105292; E. Lunghi and D. Wyler, Phys. Lett. B 521, 320 (2001) arXiv:hep-ph/0109149 . D. Becirevic et al., Nucl. Phys. B 634, 105 (2002) arXiv:hep-ph/0112303; S. Khalil and E. Kou, Phys. Rev. D 67, 055009 (2003) arXiv:hep-ph/0212023]; Phys. Rev. Lett. 91, 241602 (2003) arXiv:hep-ph/0303214 ; G. L. Kane, P. Ko, H. b. Wang, C. Kolda, J. h. Park and L. T. Wang, Phys. Rev. D 70, 035015 (2004) arXiv:hep-ph/0212092 ; Phys. Rev. Lett. 90, 141803 (2003) arXiv:hep-ph/0304239]; M. Ciuchini, E. Franco, A. Masiero and L. Silvestrini, Phys. Rev. D 67, 075016 (2003) [Erratum-ibid. D 68, 079901 (2003)] arXiv:hep-ph/0212397; K. Agashe and C. D. Carone, Phys. Rev. D 68, 035017 (2003) arXiv:hep-ph/0304229. R. Harnik, D. T. Larson, H. Murayama and A. Pierce, Phys. Rev. D 69, 094024 (2004) arXiv:hep-ph/0212180]; J. Foster, K. i. Okumura and L. Roszkowski, JHEP 0508, 094 (2005) arXiv:hep-ph/0506146;

[18] J. Foster, K. i. Okumura and L. Roszkowski, Phys. Lett. B 641, 452 (2006) arXiv:hep-ph/0604121; M. Ciuchini and L. Silvestrini, Phys. Rev. Lett. 97, 021803 (2006) arXiv:hep-ph/0603114; M. Endo and S. Mishima, Phys. Lett. B 640, 205 (2006) arXiv:hep-ph/0603251.

[19] A. Bouquet, J. Kaplan and C. A. Savoy, Phys. Lett. B 148, 69 (1984). A. Bouquet, J. Kaplan 
and C. A. Savoy, Nucl. Phys. B 262, 299 (1985).

[20] L. J. Hall, V. A. Kostelecky and S. Raby, Nucl. Phys. B 267, 415 (1986).

[21] S. Bertolini, F. Borzumati, A. Masiero, and G. Ridolfi, Nucl. Phys. B 353, 591 (1991); T. Goto, T. Nihei, and Y. Okada, Phys. Rev. D 53, 5233 (1996) [Erratum-ibid. D 54, 5904 (1996)]; T. Goto, Y. Okada, and Y. Shimizu, arXiv:hep-ph/9908499; A. Bartl, T. Gajdosik, E. Lunghi, A. Masiero, W. Porod, H. Stremnitzer, and O. Vives, Phys. Rev. D 64, 076009 (2001).

[22] S. Baek, T. Goto, Y. Okada and K.-i. Okumura, Phys. Rev. D 63, 051701 (2001) arXiv:hep-ph/0002141]; ibid. 64, 095001 (2001) arXiv:hep-ph/0104146].

[23] T. Moroi, JHEP 0003, 019 (2000) arXiv:hep-ph/0002208; T. Moroi, Phys. Lett. B 493, 366 (2000) arXiv:hep-ph/0007328.

[24] R. Barbieri, L. J. Hall and A. Strumia, Nucl. Phys. B 445, 219 (1995) arXiv:hep-ph/9501334; ibid. 449, 437 (1995) arXiv:hep-ph/9504373]; A. Masiero, M. Piai, A. Romanino and L. Silvestrini, Phys. Rev. D 64, 075005 (2001) arXiv:hep-ph/0104101]; N. Akama, Y. Kiyo, S. Komine and T. Moroi, Phys. Rev. D 64, 095012 (2001) arXiv:hep-ph/0104263; J. Hisano and Y. Shimizu, Phys. Lett. B 565, 183 (2003) arXiv:hep-ph/0303071; ibid. 581, 224 (2004) arXiv:hep-ph/0308255; D. Chang, A. Masiero and H. Murayama, Phys. Rev. D 67, 075013 (2003) arXiv:hep-ph/0205111; M. Ciuchini, A. Masiero, L. Silvestrini, S. K. Vempati and O. Vives, Phys. Rev. Lett. 92, 071801 (2004) arXiv:hep-ph/0307191; M. Ciuchini, A. Masiero, P. Paradisi, L. Silvestrini, S. K. Vempati and O. Vives, Nucl. Phys. B 783, 112 (2007) arXiv:hep-ph/0702144.

[25] L. Calibbi, A. Faccia, A. Masiero and S. K. Vempati, Phys. Rev. D 74, 116002 (2006) arXiv:hep-ph/0605139;

[26] T. Goto, Y. Okada, Y. Shimizu, T. Shindou and M. Tanaka, Phys. Rev. D 66, 035009 (2002) arXiv:hep-ph/0204081.

[27] T. Goto, Y. Okada, Y. Shimizu, T. Shindou and M. Tanaka, Phys. Rev. D 70, 035012 (2004) arXiv:hep-ph/0306093.

[28] P. Ball and R. Fleischer, Eur. Phys. J. C 48, 413 (2006) arXiv:hep-ph/0604249]; G. Isidori and P. Paradisi, Phys. Lett. B 639, 499 (2006) arXiv:hep-ph/0605012); S. Khalil, Phys. Rev. D 74, 035005 (2006) arXiv:hep-ph/0605021; S. Baek, JHEP 0609, 077 (2006) arXiv:hep-ph/0605182; R. Arnowitt, B. Dutta, B. Hu and S. Oh, Phys. Lett. B 641, 305 (2006) arXiv:hep-ph/0606130; B. Dutta and Y. Mimura, Phys. Rev. Lett. 97, 241802 
(2006) arXiv:hep-ph/0607147]; S. Nandi and J. P. Saha, Phys. Rev. D 74, 095007 (2006) arXiv:hep-ph/0608341.

[29] J. Polchinski and M. B. Wise, Phys. Lett. B 125, 393 (1983). Y. Kizukuri and N. Oshimo, Phys. Rev. D 45, 1806 (1992); ibid. 46, 3025 (1992); R. Barbieri, A. Romanino and A. Strumia, Phys. Lett. B 369, 283 (1996) arXiv:hep-ph/9511305]. T. Falk, K. A. Olive and M. Srednicki, Phys. Lett. B 354, 99 (1995) arXiv:hep-ph/9502401]; T. Falk and K. A. Olive, Phys. Lett. B 375, 196 (1996) arXiv:hep-ph/9602299; ibid. 439, 71 (1998) arXiv:hep-ph/9806236.

[30] T. Ibrahim and P. Nath, Phys. Lett. B 418, 98 (1998) arXiv:hep-ph/9707409]; Phys. Rev. D 57, 478 (1998) arXiv:hep-ph/9708456; 58, 019901(E) (1998); 60, 079903(E) (1999); 60, 119901(E) (1999); T. Goto, Y. Y. Keum, T. Nihei, Y. Okada and Y. Shimizu, Phys. Lett. B 460, 333 (1999) arXiv:hep-ph/9812369.

[31] D. Chang, W. Y. Keung and A. Pilaftsis, Phys. Rev. Lett. 82, 900 (1999) [Erratum-ibid. 83, 3972 (1999)] arXiv:hep-ph/9811202.

[32] T. Falk, K. A. Olive, M. Pospelov and R. Roiban, Nucl. Phys. B 560, 3 (1999) arXiv:hep-ph/9904393.

[33] J. Hisano and Y. Shimizu, Phys. Rev. D 70, 093001 (2004) arXiv:hep-ph/0406091; J. Hisano, M. Kakizaki, M. Nagai and Y. Shimizu, Phys. Lett. B 604, 216 (2004) arXiv:hep-ph/0407169.

[34] P. Minkowski, Phys. Lett. B 67 (1977) 421; M. Gell-Mann, P. Ramond and R. Slansky, Proceedings of the Supergravity Stony Brook Workshop, New York 1979, eds. P. Van Nieuwenhuizen and D. Freedman; T. Yanagida, Proceedinds of the Workshop on Unified Theories and Baryon Number in the Universe, Tsukuba, Japan 1979, ed.s A. Sawada and A. Sugamoto; R. N. Mohapatra and G. Senjanovic, Phys. Rev. Lett. 44 (1980) 912.

[35] B. Pontecorvo, Sov. Phys. JETP 6, 429 (1957) [Zh. Eksp. Teor. Fiz. 33, 549 (1957)]; Sov. Phys. JETP 7, 172 (1958) [Zh. Eksp. Teor. Fiz. 34, 247 (1957)]; Sov. Phys. JETP 26, 984 (1968) [Zh. Eksp. Teor. Fiz. 53, 1717 (1967)]. Z. Maki, M. Nakagawa and S. Sakata, Prog. Theor. Phys. 28, 870 (1962);

[36] Y. Farzan, Phys. Rev. D 69, 073009 (2004) arXiv:hep-ph/0310055]; Y. Farzan and M. E. Peskin, Phys. Rev. D 70, 095001 (2004) arXiv:hep-ph/0405214. Y. Farzan, JHEP 0502, 025 (2005) arXiv:hep-ph/0411358.

[37] F. Borzumati and A. Masiero, Phys. Rev. Lett. 57, 961 (1986); J. Hisano, T. Moroi, K. Tobe and M. Yamaguchi, Phys. Rev. D 53, 2442 (1996) arXiv:hep-ph/9510309; 
S. T. Petcov, S. Profumo, Y. Takanishi and C. E. Yaguna, Nucl. Phys. B 676, 453 (2004) arXiv:hep-ph/0306195.

[38] J. R. Ellis, M. K. Gaillard and D. V. Nanopoulos, Phys. Lett. B 88, 320 (1979).

[39] A. Pomarol and D. Tommasini, Nucl. Phys. B 466, 3 (1996) arXiv:hep-ph/9507462; R. Barbieri, G. R. Dvali and L. J. Hall, Phys. Lett. B 377, 76 (1996) arXiv:hep-ph/9512388; R. Barbieri and L. J. Hall, Nuovo Cim. A 110, 1 (1997) arXiv:hep-ph/9605224]; R. Barbieri, L. Giusti, L. J. Hall and A. Romanino, Nucl. Phys. B 550, 32 (1999) arXiv:hep-ph/9812239.

[40] R. Barbieri, L. J. Hall and A. Romanino, Phys. Lett. B 401, 47 (1997) arXiv:hep-ph/9702315; R. Barbieri, L. J. Hall, S. Raby and A. Romanino, Nucl. Phys. B 493, 3 (1997) arXiv:hep-ph/9610449.

[41] R. Barbieri, P. Creminelli and A. Romanino, Nucl. Phys. B 559, 17 (1999) arXiv:hep-ph/9903460; T. Blažek, S. Raby and K. Tobe, Phys. Rev. D 60, 113001 (1999) arXiv:hep-ph/9903340; ibid. 62, 055001 (2000) arXiv:hep-ph/9912482; A. Aranda, C. D. Carone and R. F. Lebed, Phys. Lett. B 474, 170 (2000) arXiv:hep-ph/9910392; Phys. Rev. D 62, 016009 (2000) arXiv:hep-ph/0002044; M. C. Chen and K. T. Mahanthappa, Phys. Rev. D 62, 113007 (2000) arXiv:hep-ph/0005292]; A. Aranda, C. D. Carone and P. Meade, Phys. Rev. D 65, 013011 (2002) arXiv:hep-ph/0109120]; S. Raby, Phys. Lett. B 561, 119 (2003) arXiv:hep-ph/0302027.

[42] S. P. Martin and M. T. Vaughn, Phys. Rev. D 50, 2282 (1994) arXiv:hep-ph/9311340. Y. Yamada, Phys. Rev. D 50, 3537 (1994) arXiv:hep-ph/9401241. I. Jack and D. R. T. Jones, Phys. Lett. B 333, 372 (1994) arXiv:hep-ph/9405233. I. Jack, D. R. T. Jones, S. P. Martin, M. T. Vaughn and Y. Yamada, Phys. Rev. D 50, 5481 (1994) arXiv:hep-ph/9407291.

[43] D. M. Pierce, J. A. Bagger, K. T. Matchev and R. j. Zhang, Nucl. Phys. B 491, 3 (1997) arXiv:hep-ph/9606211.

[44] M. Okamoto, PoS LAT2005, 013 (2006) arXiv:hep-lat/0510113; C. R. Allton et al., Phys. Lett. B 453, 30 (1999) arXiv:hep-lat/9806016]; D. Becirevic, V. Gimenez, G. Martinelli, M. Papinutto and J. Reyes, JHEP 0204, 025 (2002) arXiv:hep-lat/0110091.

[45] B. Kayser, M. Kuroda, R. D. Peccei and A. I. Sanda, Phys. Lett. B 237, 508 (1990); I. Dunietz, H. R. Quinn, A. Snyder, W. Toki and H. J. Lipkin, Phys. Rev. D 43, 2193 (1991).

[46] N. G. Deshpande, X. G. He and J. Trampetic, Phys. Lett. B 377, 161 (1996) arXiv:hep-ph/9509346; R. Barbieri and A. Strumia, Nucl. Phys. B 508, 3 (1997) 
arXiv:hep-ph/9704402]; R. Fleischer, Z. Phys. C 58, 483 (1993); ibid. 62, 81 (1994).

[47] A. L. Kagan and M. Neubert, Phys. Rev. D 58, 094012 (1998) arXiv:hep-ph/9803368.

[48] C. Greub, T. Hurth and D. Wyler, Phys. Lett. B 380, 385 (1996) arXiv:hep-ph/9602281; Phys. Rev. D 54, 3350 (1996) arXiv:hep-ph/9603404]; N. Pott, Phys. Rev. D 54, 938 (1996) arXiv:hep-ph/9512252.

[49] D. Atwood, M. Gronau and A. Soni, Phys. Rev. Lett. 79, 185 (1997) arXiv:hep-ph/9704272.

[50] S. Weinberg, Phys. Rev. Lett. 63, 2333 (1989).

[51] S. Kanemura, K. Matsuda, T. Ota, T. Shindou, E. Takasugi and K. Tsumura, Phys. Rev. D 72, 055012 (2005) arXiv:hep-ph/0507264]; 72, 059904(E) (2005); S. T. Petcov, T. Shindou and Y. Takanishi, Nucl. Phys. B 738, 219 (2006) arXiv:hep-ph/0508243; S. T. Petcov and T. Shindou, Phys. Rev. D 74, 073006 (2006) arXiv:hep-ph/0605151.

[52] A. Heister et al. [ALEPH Collaboration], Phys. Lett. B 526, 206 (2002) arXiv:hep-ex/0112011; A. Heister et al. [ALEPH Collaboration], Phys. Lett. B 583, 247 (2004); J. Abdallah et al. [DELPHI Collaboration], Eur. Phys. J. C 31, 421 (2004) arXiv:hep-ex/0311019]; P. Achard et al. [L3 Collaboration], Phys. Lett. B 580, 37 (2004) arXiv:hep-ex/0310007; G. Abbiendi et al. [OPAL Collaboration], Eur. Phys. J. C 32, 453 (2004) arXiv:hep-ex/0309014]; S. Schael et al. [The LEP Collaborations ALEPH, DELPHI, L3 and OPAL and The LEP Working Group for Higgs Boson searches], Eur. Phys. J. C 47, 547 (2006) arXiv:hep-ex/0602042]; A. A. Affolder et al. [CDF Collaboration], Phys. Rev. Lett. 88, 041801 (2002) arXiv:hep-ex/0106001; M. Martinez-Perez [CDF Collaboration], AIP Conf. Proc. 903, 189 (2007); V. M. Abazov et al. [D0 Collaboration], Phys. Lett. B 638, 119 (2006) arXiv:hep-ex/0604029.

[53] P. Koppenburg et al. [Belle Collaboration], Phys. Rev. Lett. 93, 061803 (2004) arXiv:hep-ex/0403004; B. Aubert et al. [BABAR Collaboration], Phys. Rev. D 72, 052004 (2005) arXiv:hep-ex/0508004]; S. Chen et al. [CLEO Collaboration], Phys. Rev. Lett. 87, 251807 (2001) arXiv:hep-ex/0108032.

[54] M. Ahmed et al. [MEGA Collaboration], Phys. Rev. D 65, 112002 (2002) arXiv:hep-ex/0111030.

[55] B. Aubert et al. [BABAR Collaboration], Phys. Rev. Lett. 95, 041802 (2005) arXiv:hep-ex/0502032.

[56] B. Aubert et al. [BABAR Collaboration], Phys. Rev. Lett. 96, 041801 (2006) 
arXiv:hep-ex/0508012].

[57] M. V. Romalis, W. C. Griffith and E. N. Fortson, Phys. Rev. Lett. 86, 2505 (2001) arXiv:hep-ex/0012001.

[58] C. A. Baker et al., Phys. Rev. Lett. 97, 131801 (2006) arXiv:hep-ex/0602020.

[59] B. C. Regan, E. D. Commins, C. J. Schmidt and D. DeMille, Phys. Rev. Lett. 88, 071805 (2002).

[60] E. Barberio et al. [Heavy Flavor Averaging Group (HFAG) Collaboration], arXiv:0704.3575 [hep-ex].

[61] A. Abulencia et al. [CDF Collaboration], Phys. Rev. Lett. 97, 242003 (2006) arXiv:hep-ex/0609040.

[62] J. L. Feng, K. T. Matchev and T. Moroi, Phys. Rev. D 61, 075005 (2000) arXiv:hep-ph/9909334.

[63] M. Drees and M. M. Nojiri, Phys. Rev. D 47, 376 (1993) arXiv:hep-ph/9207234; S. Mizuta and M. Yamaguchi, Phys. Lett. B 298, 120 (1993) arXiv:hep-ph/9208251]; J. Edsjö and P. Gondolo, Phys. Rev. D 56, 1879 (1997) arXiv:hep-ph/9704361. J. L. Feng, K. T. Matchev and F. Wilczek, Phys. Lett. B 482, 388 (2000) arXiv:hep-ph/0004043];

[64] J. R. Ellis, T. Falk and K. A. Olive, Phys. Lett. B 444, 367 (1998) arXiv:hep-ph/9810360.

[65] T. E. Browder, M. Ciuchini, T. Gershon, M. Hazumi, T. Hurth, Y. Okada and A. Stocchi, arXiv:0710.3799 [hep-ph].

[66] B. Grinstein, Y. Grossman, Z. Ligeti and D. Pirjol, Phys. Rev. D 71, 011504 (2005) arXiv:hep-ph/0412019. M. Matsumori and A. I. Sanda, Phys. Rev. D 73, 114022 (2006) arXiv:hep-ph/0512175]. P. Ball and R. Zwicky, Phys. Lett. B 642, 478 (2006) arXiv:hep-ph/0609037.

[67] Y. Grossman, G. Isidori and M. P. Worah, Phys. Rev. D 58, 057504 (1998) arXiv:hep-ph/9708305. M. Beneke, Phys. Lett. B 620, 143 (2005) arXiv:hep-ph/0505075]. A. R. Williamson and J. Zupan, Phys. Rev. D 74, 014003 (2006) [Erratum-ibid. D 74, 03901 (2006)] arXiv:hep-ph/0601214.

[68] L. J. Hall, R. Rattazzi and U. Sarid, Phys. Rev. D 50, 7048 (1994) arXiv:hep-ph/9306309. K. S. Babu and C. F. Kolda, Phys. Lett. B 451, 77 (1999) arXiv:hep-ph/9811308. M. S. Carena, D. Garcia, U. Nierste and C. E. M. Wagner, Nucl. Phys. B 577, 88 (2000) arXiv:hep-ph/9912516. 
[69] C. S. Huang, W. Liao and Q. S. Yan, Phys. Rev. D 59, 011701 (1999) arXiv:hep-ph/9803460;

C. Hamzaoui, M. Pospelov and M. Toharia, Phys. Rev. D 59, 095005 (1999) arXiv:hep-ph/9807350; S. R. Choudhury and N. Gaur, Phys. Lett. B 451, 86 (1999) arXiv:hep-ph/9810307]; K. S. Babu and C. F. Kolda, Phys. Rev. Lett. 84, 228 (2000) arXiv:hep-ph/9909476; 